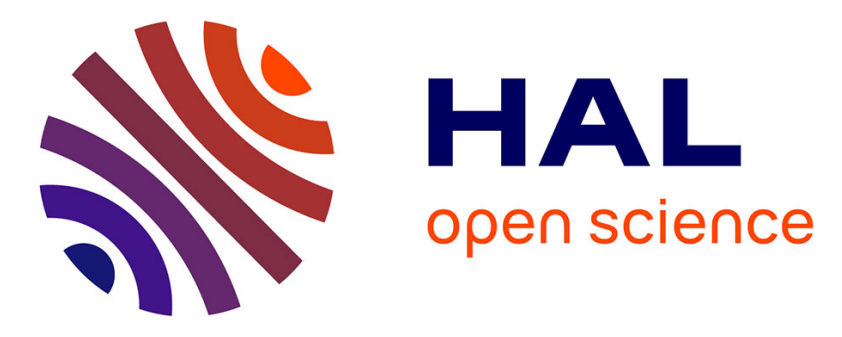

\title{
Linking plastic deformation to recrystallization in metals, using digital microstructures
}

Roland E. Logé, Marc Bernacki, Héba Resk, Laurent Delannay, Hugues

Digonnet, Yvan Chastel, Thierry Coupez

\section{- To cite this version:}

Roland E. Logé, Marc Bernacki, Héba Resk, Laurent Delannay, Hugues Digonnet, et al.. Linking plastic deformation to recrystallization in metals, using digital microstructures. Philosophical Magazine, 2009, 88 (30-32), pp.3691-3712. 10.1080/14786430802502575 . hal-00513982

\section{HAL Id: hal-00513982 \\ https://hal.science/hal-00513982}

Submitted on 1 Sep 2010

HAL is a multi-disciplinary open access archive for the deposit and dissemination of scientific research documents, whether they are published or not. The documents may come from teaching and research institutions in France or abroad, or from public or private research centers.
L'archive ouverte pluridisciplinaire $\mathbf{H A L}$, est destinée au dépôt et à la diffusion de documents scientifiques de niveau recherche, publiés ou non, émanant des établissements d'enseignement et de recherche français ou étrangers, des laboratoires publics ou privés. 


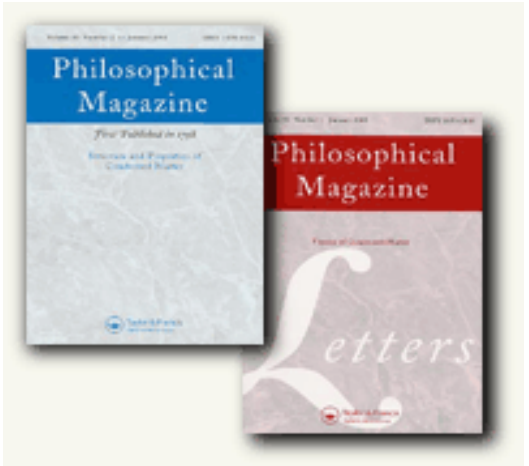

Linking plastic deformation to recrystallization in metals, using digital microstructures

\begin{tabular}{|c|c|}
\hline Journal: & Philosophical Magazine \& Philosophical Magazine Letters \\
\hline Manuscript ID: & TPHM-08-Jan-0006.R1 \\
\hline Journal Selection: & Philosophical Magazine \\
\hline $\begin{array}{r}\text { Date Submitted by the } \\
\text { Author: }\end{array}$ & 16-Sep-2008 \\
\hline Complete List of Authors: & $\begin{array}{l}\text { Loge, Roland; Ecole des Mines de Paris, CEMEF } \\
\text { Bernacki, Marc; Ecole des Mines de Paris, CEMEF } \\
\text { Resk, Heba; Ecole des Mines de Paris, CEMEF } \\
\text { Delannay, Laurent; UCL, MEMA } \\
\text { Digonnet, Hugues; Ecole des Mines de Paris, CEMEF } \\
\text { Chastel, Yvan; Ecole des Mines de Paris, CEMEF } \\
\text { Coupez, Thierry; Ecole des Mines de Paris, CEMEF }\end{array}$ \\
\hline Keywords: & $\begin{array}{l}\text { finite-element modelling, grain boundaries, grain growth, } \\
\text { microstructure, nucleation, plasticity, recrystallization }\end{array}$ \\
\hline Keywords (user supplied): & level set \\
\hline
\end{tabular}

\section{今 ScholarONE" \\ Manuscript Central}


Linking plastic deformation to recrystallization in metals, using digital microstructures

Roland Logéa ${ }^{a *}$, Marc Bernacki $^{\mathrm{a}}$, Heba Resk $^{\mathrm{a}}$, Laurent Delannay ${ }^{\mathrm{b}}$, Hugues Digonnet ${ }^{\mathrm{a}}$, Yvan Chastel $^{\mathrm{a}}$, Thierry Coupez ${ }^{\mathrm{a}}$

${ }^{a}$ MINES ParisTech, CEMEF - Center of Materials Forming, CNRS UMR 7635, BP 207, 06904 Sophia Antipolis Cedex, France

${ }^{b}$ CESAME - MEMA, Université catholique de Louvain (UCL), bâtiment Euler, av. G. Lemaître 4, 1348 Louvain-laNeuve, Belgium

Abstract

Procedures for synthesizing digital polycrystalline microstructures are illustrated, either from 2D statistical data or from 3D deterministic data. Finite element meshes representing the digital microstructures are generated using anisotropic and adaptive mesh refinement close to the grain boundaries. Digital mechanical testing based on crystal plasticity theory provides an estimate of the spatial distribution of strain energy within the polycrystalline aggregate. The latter quantity is used as an input for modelling subsequent static recrystallization, grain boundary motion being described within a level set framework. The kinetic law for interface motion accounts for both the stored strain energy and the grain boundary energy. The possibility to include nucleation events within the level set framework is illustrated, as well as the evolving topology of the grain boundary network. The recrystallization model is tested in different configurations and compared to the Johnson-MehlAvrami-Kolmogorov (JMAK) theory.

Keywords: digital microstructure; finite elements; meshing; remeshing; crystal plasticity; large deformation; recrystallization; level set; moving boundaries; nucleation, JMAK theory.

\section{Introduction}

No generic model is currently available to describe the recrystallization behavior of a wide class of metallic alloys. This is due in great measure to the fact that recrystallization, like many similar state-altering processes that are driven by thermal energy and internal energy of structural defects, is really controlled by factors at several length scales. Multiscale modelling is thus a requisite to coming to a better understanding of recrytallization. Important factors over length scales of decreasing size include: (a) grain interactions, which set up stress fields that persist over grain

\footnotetext{
* Corresponding author. Email: Roland.Loge@ mines-paristech.fr
} 
dimensions, (b) stress variations within grains due to the presence of dislocation structures, which both perturb the longer range field across the grain and impact the mobility of interfaces through grains, (c) energies and mobilities of interfaces, which control their kinetics, and (d) particles and solutes, interacting with interfaces and modifying their kinetics.

Over the last decade, considerable progress has been made in the numerical simulation of recrystallization phenomena [1]. Common approaches include the Monte Carlo (MC) method [2], the Cellular Automaton (CA) methods [2,3], the phase field method [4] and the level set method [5]. The first two are probabilistic techniques which deliver evolving grain structures. They are associated with a $2 \mathrm{D}$ or $3 \mathrm{D}$ geometric representation of the microstructure, discretized on a regular grid made of "cells" which are allocated to the grains.

The standard MC method as derived from the Potts model (multistate Ising model) applies probabilistic rules in order to update each cell at each time step of the simulation. The use of this model in 3D is relatively easy and efficient [6]. However the comparison between MC results and experiments is not straightforward [7]. Furthermore, the standard form of the model does not result in a linear relationship between stored energy and migration rate. The CA method, on the other hand, uses physically based rules to determine the rate at which a transformation front propagates across neighbouring cells [3], and can therefore be related to the microstructure and kinetics of a real system. In the case of primary recrystallization the switch rule is simple: an unrecrystallized cell switches to the recrystallized state if one of its neighbours is already recrystallized. A major drawback of the CA method is the absence of appropriate method to treat nucleation phenomena [7].

The two others methods, i.e. the phase field and level set methods, have many common points. They both have the advantage of avoiding numerical difficulties related to interface tracking. The principle of the phase-field model consists in describing the location of phases by introducing an order parameter (the phase field) which varies smoothly from one to zero (or minus one to one) through a diffuse interface [8]. The concept has been extended to deal with more complex problems involving more than two phases and also to model microstructure evolution $[9,10]$. As for the MC or CA methods, the topological events are treated in a natural way as a result of energy minimization. In the case of 2D ideal normal grain growth, published results illustrate the potential of the approach [9]. However, the main difficulty of this method remains the construction of the free energy density function. Furthermore, the energy minimization of each 
order parameter can involve very expensive and intensive calculations, particularly for three-dimensional systems [11]. In contrast, the level set method [12,13] is now commonly used to follow propagating fronts in various models [14]. The level set method has been extended to model the motion of multiple junctions when more than two regions or grains intersect [15].

\section{Methodology}

This paper proposes a first step in the direction of multiscale modelling of recrystallization in polycrystalline metals, by considering digital microstructures [16-18]. Representative digital microstructures are synthesized with a specific software, based on the construction of Voronoï cells. The distribution of grain sizes and grain shapes is monitored and adapted according to experimental data. In the same way, attributes of grains, such as crystallographic orientation, are sampled so that global properties (e.g. crystallographic texture) are well reproduced. The digital representation of the microstructure is discretized into a finite element mesh, which is then used to model plastic deformation and subsequent recrystallization. The local behaviour of individual microstructure components may be computed through models operating at different scales. For example, grain constitutive models may be derived from the modelling of dislocation populations within the grains, and grain boundary motion may be described by connecting the continuum mechanical and thermal fields to simulations at the atomistic and dislocations levels. Validation of this "digital microstructure" approach is typically done using in situ experiments, e.g. synchrotron measurements, where the evolving microstructure can be characterized in 3D before, during and after thermo-mechanical processing.

Previous attempts to use digital microstructures for the modelling of plastic deformation and subsequent static recrystallization have been reported by few authors. In [19], a 2D digital sample was meshed, mechanical testing was performed with crystal plasticity finite element simulations, and thermal treatment involving recrystallization was done subsequently using a CA approach [19]. A Monte Carlo (MC) approach can replace the CA approach, as done in [2023]. In general, the authors do not guarantee that the digital microstructure is representative of a real microstructure because the total number of grains being considered is small. Finite element simulation of plastic deformation is done within updated Lagrangian schemes, which imply that the deformation remains limited in order to avoid that the mesh 
degenerates [24,25], as the use of an automatic remeshing procedure is not reported. On the other hand, the finite element mesh needs to be converted into appropriate "voxel" grids for using CA or MC approaches. In doing so, some details of the microstructures may be lost, e.g. those related to grain boundary curvature. This aspect is important if secondary recrystallization is to be modelled after primary recrystallization. Furthermore, in some cases it is useful to simulate further deformation of the digital aggregate following partial recrystallization, e.g. when studying multi-pass (industrial) processing. Transforming the voxel structure back to the finite element mesh then implies a new loss of information (boundary curvature, but also distribution of residual strains/stresses, etc.).

In the approach presented here, there is only one conversion of the initial digital aggregate into a finite element mesh. The digital aggregate can be obtained from experiments, from other simulations, or from a numerical construction. The format is either a Voronoï tessellation or a voxel structure. Discretization into a finite element mesh is done iteratively, by considering the position of the elements with respect to microstructure interfaces (grain boundaries in particular). "Distance functions" updated throughout the deformation simulation allow keeping track of the boundaries. The distance functions uniquely define the topology of the aggregate and the corresponding allocation of properties, even when automatic remeshing operates. The digital heat treatment is performed directly on the finite element mesh using a level set approach. The level set method consists in evolving each distance function using a convection equation, thereby implicitly updating the position of moving interfaces. The velocity of the boundaries is described by a kinetic law taking account of the bulk strain energy and the grain boundary energy, both being related to local crystallographic orientations. When describing primary recrystallization, the influence of the grain boundary energy is neglected with respect to that of the strain energy. The level set approach allows working directly on the deformed mesh, without loosing/distorting any information. The stored energy in particular is directly available and dictates the kinetics of microstructure evolution, with no need to construct a free energy density function (as in phase field methods). Nucleation phenomena are furthermore easy to implement.

The paper is organized as follows. Section 3 explains how distance functions, within a level set framework, can be used for adaptive meshing and remeshing of the microstructure during mechanical testing and subsequent heat treatment. Section 4 presents the construction of digital microstructures and the anisotropic (re-)meshing procedures providing a non uniform mesh size related to the distance functions. In Section 5, mechanical testing is simulated using crystal 
plasticity theory. Finally, in Section 6, the level set framework is presented and illustrated with 2D and 3D studies of recrystallization driven by the stored energy. The possibility to nucleate new grains in the context of primary recrystallization is demonstrated as well. Different test cases are discussed with reference to the classical Johnson-MehlAvrami-Kolmogorov (JMAK) model.

\section{Distance functions and level set framework}

A function $\phi$, defined in a domain $\Omega$, is called level set if it corresponds, at any points of $\Omega$, to the distance $d$ to an interface $\Gamma$. In other words, the interface $\Gamma$ is given by the zero level of the function $\phi$ :

$$
\left\{\begin{array}{l}
\phi(\mathbf{x})=d(\mathbf{x}, \Gamma), \mathbf{x} \in \Omega \\
\Gamma=\{\mathbf{x} \in \Omega, \phi(\mathbf{x})=0\}
\end{array}\right.
$$

When dealing with a polycrystalline aggregate, an distinct level set function is used for each grain : $\left\{\phi_{i}, 1 \leq i \leq N_{G}\right\}$, with $N_{G}$ the total number of grains in the aggregate. The chosen sign convention is $\phi_{i} \geq 0$ inside the grain $G_{i}$, and $\phi_{i} \leq 0$ outside. Figure 1 illustrates a digital sample made of fifty grains, highlighting four particular grains of the microstructure which are displayed using the zero levels of the corresponding level set functions.

When interfaces propagate with a known velocity $\xi(\mathbf{x})$ in $\Omega$, the values of distance functions are updated using a convection equation :

$$
\left\{\begin{array}{l}
\frac{\partial \phi_{i}}{\partial t}+\xi \cdot \nabla \phi_{i}=0 \\
\phi_{i}(t, \mathbf{x}=0)=\phi_{i}^{0}(\mathbf{x})
\end{array} \quad \forall i \in\left\{1, \ldots, N_{G}\right\} .\right.
$$

Solving (2) then implicitly updates the position of moving interfaces as a function of time. The method "captures" the interface, rather than tracking it (e.g. see vertex models [27]). Periodic re-initialization of the solution is needed in order to preserve the property of a distance function [15,28].

The level set method is particularly appropriate for handling complex topological events such as the disappearance of grains; no special treatment is needed. A second advantage comes from the fact that intrinsic geometric properties of the front are easily determined from the values of the distance function. Indeed, at any point of the front: 


$$
\mathbf{n}=\nabla \phi /\|\nabla \phi\|, \quad \kappa=-\nabla \cdot \mathbf{n}
$$

where $\mathbf{n}$ is a unit vector normal to the interface, which indicates the direction of propagation, and $\kappa$ the curvature of the interface. The latter quantity is useful when dealing with secondary recrystallization.

\section{Automatic meshing of the digital microstructure}

2D or 3D polycrystalline microstructures are numerically generated using either a recursive Voronoï tesselation algorithm or an existing pixel/voxel based representation. These microstructures can be optimized according to desired criteria of sizes and shape, as discussed in [29].

Figure 2 illustrates how a 3D initial finite element mesh can be constructed from either a Voronoï structure or a voxel structure. Both Voronoï cells and voxels are easy to divide into tetrahedral elements. In the former case, the obtained mesh is very coarse and will need to be refined. In the latter case, the mesh is on the contrary too fine, and needs to be coarsened. Appropriate refinement of the mesh along interfaces such as grain boundaries is useful to capture the large strain rate/stress gradients developing across those interfaces upon deformation of the microstructure. Such gradients result from the heterogeneous mechanical response of neighbouring grains induced by the crystallographic orientations.

Figure 3 presents a Voronoï tessellation made of 50 grains, and the corresponding finite element mesh, made of tetrahedral elements with heterogeneous sizes and shapes. Anisotropic meshing is used close to the grain boundaries, with a smaller element size in the direction perpendicular to the boundary. A corresponding anisotropic metric is defined, while an isotropic metric is applied in the bulk of the grains. A metric is a symmetric positive tensor $\mathbf{M}$ used to measure the scalar product of two vectors in a stretched, local base :

$$
<\mathbf{x}, \mathbf{y}>_{\mathbf{M}}={ }^{t} \mathbf{x M y} \text {. }
$$

If $\mathbf{M}$ is the identity tensor, the scalar product corresponds to the usual one in the Euclidian space.

Anisotropic meshes are built using the MTC algorithm developed by Coupez [30,31]. Mesh generation is topological, in the sense that mesh topology is iteratively improved by considering the quality of the elements. The latter is defined by assigning the highest quality to the elements with all edges of the same length, and the worst quality to elements which 
tend to degenerate from a volume to a surface (in 3D), or from a surface to a segment (in 2D). The shape factor $c(e)$ characterizing the quality is normalized within the interval $[0,1]$ :

$$
c(e)=c_{0} \frac{|e|}{l(e)^{k}},
$$

where $c_{0}$ is a normalisation coefficient, $|e|$ the volume of the element, $l(e)$ the average length of the element edges and $k$ the space dimension. The volume and lengths are computed with the modified scalar product (Equation 4) when anisotropic meshing is needed. Hence, the MTC algorithm improves the topology of the elements by raising the shape factor of the elements that define this topology.

The definition of the metric field leads to a non-uniform, anisotropic mesh, which is refined close to the grain boundaries. The metric $\mathbf{M}$ is a tensor whose eigenvalues and eigenvectors define the transformation of an isotropic mesh of size $h$ into an anisotropic mesh with a directional dependence of $h$. This can be illustrated with a simple configuration of two grains separated by one interface. The direction of mesh refinement is the normal to the interface. To specify the mesh size in that direction, as well as the evolution of mesh size in space, a characteristic length $l_{0}$ is introduced. When the distance function is smaller than $l_{0}$, the corresponding mesh is considered to be "close" to the interface :

$$
\left\{\begin{array}{l}
|\phi(\mathbf{x})|>l_{0} \quad \text { far from the interface } \\
|\phi(\mathbf{x})|<l_{0} \quad \text { near the interface }
\end{array} .\right.
$$

The mesh size takes a default value far from the interface, and is reduced in the direction perpendicular to the interface when $|\phi|$ is reduced. For example, the mesh size $h$ may be chosen as

$$
\left\{\begin{array}{l}
|\phi(\mathbf{x})|>l_{0} \quad \Rightarrow h=h_{d} \\
|\phi(\mathbf{x})|<l_{0} \Rightarrow h=\frac{h_{d}(m-1)}{m l_{0}}|\phi(\mathbf{x})|+\frac{h_{d}}{m}
\end{array}\right.
$$

At the interface the mesh size is reduced by a factor $m$ with respect to the default value $h_{d}$. This mesh size then increases steadily to reach the default value $h_{d}$ at the distance $l_{0}$. Such variations in mesh size are directly implemented by varying the corresponding metric $\mathbf{M}$.

When dealing with polycrystalline aggregates and multiple interfaces, the above strategy is repeated for each grain. For the nodes at which 


$$
\left|\phi_{i}(\mathbf{x})\right|>l_{0}, \quad 1 \leq i \leq N_{G},
$$

there is no direction of refinement, and the mesh is isotropic with $h=h_{d}$. On the contrary, there may be several directions of refinement when there is more than one level set function for which $\left|\phi_{i}(\mathbf{x})\right|<l_{0}$, and when the corresponding normal directions $\mathbf{n}_{\mathrm{i}}$ calculated from (3), are not co-linear. These independent vectors are the eigenvectors of the metric defined in (4). For each vector direction the eigenvalue is calculated from the mesh size prescribed in (7), with $\phi(\mathbf{x})$ being the level set function associated to the considered normal. At triple or multiple junctions, the refinement may therefore become isotropic.

Figure 4 illustrates the method which has been applied to a digital microstructure made of one thousand grains. The mesh (2 125688 nodes, 12385889 elements) was obtained after a 26 hours parallel computation on 24 processors of a 98 cores Opteron 280 2,4GHz linux cluster. The refinement of the mesh may become significant when approaching grain boundaries, as already observed in Figure 3 (see enlarged section). In the direction normal to the interface, the length scale is one at which mechanical behaviour may be influenced by the discrete nature of dislocation motion. Nevertheless, traditional crystal plasticity theory is used here (Section 5), thus neglecting some stress concentrators and their potential effect on recrystallisation. Even is this simplified situation, the stored energy field induced by plastic deformation is particularly heterogeneous at the grain boundaries. Besides, strong refinement is needed for the modelling of grain boundary motion in the recrystallization regime. Indeed, in the level set approach (Section 3), the accuracy of the geometric properties of the boundaries, defined by Equations (3), is a direct consequence of the mesh size (see more details in Section 6.1).

\section{Digital mechanical testing under large deformations}

Mechanical testing is performed using finite element simulations where each integration point of the mesh behaves as a single crystal subjected to finite strain increments. The finite element approach is based on a mixed velocity-pressure formulation with an enhanced (P1+/P1) 4-node tetrahedral element [32]. Classical theory of crystal plasticity [33-36] is 
considered, using a slightly modified version of the time integration algorithm developed by Delannay et al. [37]. For computational efficiency, one computes rates of lattice rotation and rates of dislocation slip in a decoupled way.

\subsection{Elastic-viscoplastic crystal constitutive law}

The main equations described in [37] are briefly recalled. The elastic-viscoplastic formulation is based on a multiplicative decomposition of the deformation gradient tensor, $\mathbf{F}=\mathbf{R}^{*} \mathbf{U}^{e l} \mathbf{F}^{\mathrm{p}}$, where $\mathbf{R}^{*}, \mathbf{U}^{e l}$ and $\mathbf{F}^{\mathrm{p}}$ represent, respectively, the rotation of the crystal lattice, the elastic stretch and the deformation by dislocation slip. Under the assumption of infinitesimal elastic strains, i.e. $\mathbf{U}^{e l}=\mathbf{I}+\boldsymbol{\varepsilon}^{e l}$ where $\boldsymbol{\varepsilon}^{e l}$ is symmetric and $\left\|\varepsilon^{e l}\right\|<1$, the velocity gradient tensor $\mathbf{L}=\nabla \mathbf{v}$ is closely approximated by:

$$
\mathbf{L} \cong \dot{\mathbf{R}}^{*} \mathbf{R}^{* T}+\mathbf{R}^{*}\left(\dot{\boldsymbol{\varepsilon}}^{e l}+\sum_{\alpha} \mathbf{M}^{\alpha} \dot{\gamma}^{\alpha}\right) \mathbf{R}^{* T}
$$

where $\mathbf{M}^{\alpha}$ is the Schmid tensor of slip system $\alpha$ and $\dot{\gamma}^{\alpha}$ the corresponding rate of dislocation slip. Denoting $C$ the anisotropic, fourth-order, elasticity operator, one derives the Cauchy stress tensor $\sigma$ from:

$$
\boldsymbol{\sigma}=\mathbf{R}^{*} \mathbf{T R}^{* \mathrm{~T}} \quad \text { and } \quad \dot{\mathbf{T}}=\mathrm{C} \dot{\boldsymbol{\varepsilon}}^{e l}
$$

In order to avoid ambiguity in determining the slip rates [38], the viscoplastic expression introduced by [39] is adopted :

$$
\begin{aligned}
& \dot{\gamma}^{\alpha}=\dot{\gamma}_{0}\left|\frac{\tau^{\alpha}}{\tau_{c}}\right|^{1 / m} \operatorname{sign}\left(\tau^{\alpha}\right) \\
& \text { with } \quad \tau^{\alpha}=\mathbf{T}: \mathbf{M}^{\alpha}, \\
& \tau_{c}=\tau_{c 0}\left(1+\frac{\Gamma_{t o t}}{\Gamma_{0}}\right)^{n}, \\
& \Gamma_{t o t}=\int_{0}^{t} \sum_{\alpha}\left|\dot{\gamma}^{\alpha}\right| d t .
\end{aligned}
$$

In (11), $\dot{\gamma}_{0}$ is a reference slip rate, $m$ the sensitivity exponent, and $\tau_{c}$ the critical resolved shear stress, which evolves with strain according to the hardening law (13), $\tau_{c 0}, \Gamma_{0}$ and $n$ being material parameters. 


\subsection{Time integration of the constitutive law in the finite element model}

Details of the finite element formulation are available elsewhere [40,32], only the main features are recalled here. The updated Lagrangian scheme is based on a mixed velocity-pressure formulation with an enhanced (P1+/P1) 4-node tetrahedral element. The non linear discretized finite element system is solved within a Newton-Raphson scheme using a preconditioned conjugate residual approach. Unilateral contact with the 'tools' (forming processes context) is treated with a penalty method. In the present study, the average deformation of the digital microstructure, constituting a volume element $\Omega$, is controlled by prescribing the velocity of all nodes lying on the outer boundary $\Sigma$ of $\Omega$ :

$$
\mathbf{v}=\overline{\mathbf{L}} \mathbf{x}
$$

where $\mathbf{x}$ represent the node coordinates at time $t$, and $\mathbf{v}$ the velocity that is prescribed.

The principle of virtual work is applied in the deformed configuration $\Omega$ at time $t$. Appropriate conditions are prescribed at the boundary $\Sigma$ (see above). If $\tilde{\mathbf{v}}$ denotes a kinematically admissible (virtual) velocity field and $\tilde{p}$ a virtual pressure field, one writes:

$$
\begin{aligned}
& \forall \tilde{\mathbf{v}}, \int_{\Omega}\left(\boldsymbol{\sigma}^{\prime}: \nabla \tilde{\mathbf{v}}\right) d \Omega-\int_{\Omega} p(\nabla \cdot \tilde{\mathbf{v}}) d \Omega-\int_{\Sigma}(\mathbf{t} \cdot \tilde{\mathbf{v}}) d \Sigma=0 \\
& \forall \tilde{p}, \int_{\Omega} \tilde{p}\left(\nabla \cdot \mathbf{v}+\frac{\dot{p}}{K}\right) d \Omega=0
\end{aligned}
$$

where $\sigma^{\prime}$ is the deviatoric part of the Cauchy stress $\sigma, p=-\operatorname{tr}(\boldsymbol{\sigma}) / 3$ is the pressure, $K$ the (elastic) bulk modulus, and $\mathbf{t}$ the tension applied to the boundary. While (16) and (17) are solved at time $t, \sigma$ is evaluated at time $t+\Delta t$ [40], and obtained by implicit time integration of Eqs. 9-14, using :

$$
\boldsymbol{\sigma}_{t+\Delta t}=\left.\left.\mathbf{R}^{*}\right|_{t+\Delta t}\left(\left.\mathbf{T}\right|_{t}+\left.\dot{\mathbf{T}}\right|_{t+\Delta t} \Delta t\right) \mathbf{R}^{* T}\right|_{t+\Delta t}
$$


The Newton-Raphson procedure used to solve the finite element problem relies on an algorithmic tangent operator as detailed in [37]. Once a converged velocity field $\mathbf{v}$ is obtained at time $t$, the geometry of the mesh is updated for the next time increment according to : $\left.\mathbf{x}\right|_{t+\Delta t}=\left.\mathbf{x}\right|_{t}+\mathbf{v} \Delta t \quad[40]$.

\subsection{Test case}

The objective of the test case is to analyze the spatial distribution of stored strain energy in a digital aggregate, subjected to large deformations. A channel die test has been chosen, and is illustrated in Figure 5.

The digital microstructure is made of $N_{G}=50$ grains. Slip is assumed to operate on the $12\{111\}<110>$ slip systems as is typically considered in FCC crystals at room temperature. A 35\% reduction in height is applied, and the stored energy is computed from :

$$
E=\delta \int \boldsymbol{\sigma}: \nabla \mathbf{v} d t
$$

where $\delta$ defines the fraction of the strain energy which is stored in the material, i.e. not dissipated into heat. More accurate measures of stored energy could be implemented in the future, by relying on the dislocation density computed within the crystal plasticity approach, e.g. [21,22]. Another simple approach would consist in describing both $\tau_{c}$ (see Equation (13)) and $E$ as a function of the dislocation density $\rho[41]$ :

$$
\begin{aligned}
& \tau_{c}=\tau_{c 0}+\frac{1}{2} G b \sqrt{\rho}, \\
& E=\frac{1}{2} \rho G b^{2}
\end{aligned}
$$

where $\mathrm{G}$ is the shear modulus of the material. Combining (25) and (26) leads to

$$
E=\frac{2}{G}\left(\tau_{c}-\tau_{c 0}\right)^{2}
$$


The absolute values of $E$ do not always matter, e.g. only the relative values will play a role in determining the topological evolution of the grain boundary network (see the next section). In those cases, the exact values of $G$ and $\delta$ in (27) and (24) do not need to be known.

Figure 6 shows the 3D distribution of the stored energy $E$, and of the gradient of the stored energy $\operatorname{grad}(E)$. The highest values of $E$ and $\operatorname{grad}(E)$ usually appear at grain boundaries. The latter are identified by the zero level of the $\left\{\phi_{i}, 1 \leq i \leq N_{G}\right\}$ functions, as defined in expression (1). One can also notice significant intergranular and intragranular variations of $E$, which result in maxima of $\operatorname{grad}(E)$ mainly at grain boundaries, but also inside some grains. Inversely, most grain boundaries correspond to maxima of $\operatorname{grad}(E)$, but not all of them.

These distributions are the consequence of (a) local crystallographic orientations and (b) grain interactions, i.e. neighbourhood effects. They can be used as an input for the modelling of primary recrystallization, keeping in mind that traditional continuum crystal plasticity obviously needs to be enriched, e.g. if nuclei positions are to be predicted. Constitutive models developed at lower scales or considering Geometrically Necessary Dislocations (GNDs) would be required to better reproduce local features (shear bands, twin bands, dislocation pile ups, etc.) at the origin of nucleation events. This work is only a first step in that direction.

\section{Digital heat treatment leading to recrystallization}

In this section grain boundary motion is modelled using the level set framework described in Section 3. Motion is assumed to be due to the difference in stored energy on either side of the interface, as is the case in primary recrystallization, or when Strain Induced Grain Boundary Migration (SIBM) occurs. When dealing with primary recrystallization, nucleation of new grains need to be modelled as well. Nuclei grow spontaneously since we assume that they have zero stored energy. Position and time of appearance of each new nucleus can be based on different criteria. Let us assume, for simplicity, that they appear in regions with high stored energy, or high gradients of stored energy [42]. A positioning criterion based on local crystallographic misorientations could be defined as well [43], since a nucleus will only grow if the mobility of the associated grain boundaries is high enough. This is usually true for 
misorientations larger than $10-15^{\circ}$. Time of appearance of a nucleus is often described by a probability law [44], unless site-saturated conditions are assumed. In the latter case all potential nuclei grow from $t=0$, i.e. there is no incubation time.

In the simulations described below (Section 6.3), 1000 positions of nuclei are either selected at random or correspond to regions with the highest gradients of stored energy $\|\operatorname{grad}(E)\|$. The probability of appearance at each time increment is constant and relatively low $\left(2 \cdot 10^{-4}\right)$. Grain boundary motion is related to the difference in stored energy $E$ across the boundary. For that purpose only (and not for the positioning of the nuclei), a constant value of $E$ is considered in each grain.

\subsection{Kinetic law for grain boundary motion}

Grain boundary motion is related to a driving force per unit area $\Delta f$, and the corresponding velocity $\xi$ defined in (2) is assumed to follow the kinetic relation [42] :

$$
\xi=m \Delta f \mathbf{n}
$$

where $m$ corresponds to the grain boundary mobility, and $\mathbf{n}$ to the outside unit normal to the boundary. The mobility is very often written as

$$
m=m_{0} \exp (Q / R T)
$$

and it is sensitive to the impurity (solute atom) content in the metal, as well as to the crystallographic nature of the boundary. The driving force is defined by

$$
\Delta f=\Delta E-\gamma \kappa,
$$

where $\Delta E$ is the stored energy difference across the boundary (e.g. according to (24) or (27)), $\gamma$ the boundary energy, and $\kappa$ the boundary curvature. It is assumed that the material is highly deformed before it undergoes primary recrystallization. The second term of equation (30), related to the grain boundary energy, is therefore neglected compared to the first term, related to stored strain energy. 
Interface motion using level set methods is now of common use [12]. However, in most cases, a single interface is captured, separating only two regions. In [15], the authors have extended the method to the case of multiple junctions, and simulated 2D grain growth due to grain boundary energy only. Each region has its own private level set function, and local normal velocities are defined by the nearest interface. A reassignment step is used to avoid the development of voids and overlapping regions. In a subsequent paper [5], the authors propose to add a constraint to the overall problem which imposes that the addition of all individual surfaces must correspond to the total surface of the domain.

In the present study a new formulation is used, in which (a) bulk stored energies can be taken into account, and (b) the development of voids and overlapping regions is avoided. Individual level set functions are defined for each region, as apparent in (2), and re-initialization steps [12] are periodically performed for each of them as they evolve according to the convection equation [45].

To prevent the development of overlapping regions and/or voids, all meeting interfaces described by distinct level set functions must be assigned the same velocity, i.e. the zero level of the $\phi_{i}(\mathbf{x})$ functions have to match perfectly. Consequently, the normal $\mathbf{n}$ (and also the curvature $\kappa$ if considered) to the interface must be computed very accurately from (3), for all $\phi_{i}(\mathbf{x})$ functions. This in turn requires that the mesh is strongly refined locally, close to the interfaces, compared to the bulk of the grains. To avoid the explosion of computational effort, the construction of an anisotropic mesh is required, with the methods discussed in Section 4.

The common velocity at triple or multiple junctions is of particular interest and must be defined according to the kinetic law (28) which only considers a single interface. Figure 7 shows that the direct use of (28) leads to discontinuities of the velocity field at triple junctions, which in turn leads to the development of overlapping regions and/or voids. A smoothing technique is therefore applied (Figure 8), which consists in using the following algorithm : 


$$
\begin{aligned}
& \mathbf{x} \in G_{i} \Leftrightarrow \phi_{i}(t, \mathbf{x})=\max _{1 \leq p \leq N_{G}}\left(\phi_{p}(t, \mathbf{x})\right) \\
& \Rightarrow \xi(t, \mathbf{x})=\sum_{\substack{j=1 \\
j \neq i}}^{N_{G}} \exp \left(-\eta\left|\phi_{j}(t, \mathbf{x})\right|\right) m_{i j}\left(E_{i}-E_{j}\right) \mathbf{n}_{j}(t, \mathbf{x})
\end{aligned}
$$

where $G_{i}$ designates the grain of index i, $\forall i \in\left\{1, \ldots, N_{G}\right\}, \eta$ a positive fixed parameter calibrated to obtain a negligible exponential term outside the anisotropic part of the mesh, $m_{i j}$ the mobility defined in (29) assuming an interface between grains $\mathrm{G}_{\mathrm{i}}$ and $\mathrm{G}_{\mathrm{j}}$, and $\mathbf{n}_{j}$ the outward unit normal at $\mathbf{x}$, calculated from (3) using $\phi=\phi_{j}(t, \mathbf{x})$. In Figure 8 it is observed that the smoothing technique is not sufficient by itself, it needs to be combined with anisotropic meshing to improve accuracy. The approach is validated in $2 \mathrm{D}$ in [45] by comparison with an exact solution of the triple junction problem [46], and when using anisotropic automatic remeshing every few increments. Figure 9 shows that, except for the computational efforts, no specific development is required to extend the above approach in 3D. Finally, Figure 10 illustrates a more complex microstructure evolution in $2 \mathrm{D}$, where grains with the highest $E$ disappear due to the growth of neighbouring grains.

\subsection{Nucleation of new grains}

Figure 10 has shown that when using front capturing methods for describing interface motion, there is no specific treatment to be used when some regions (grains) disappear. Complex topological evolutions are handled automatically. In a similar way, it is possible to introduce new regions (grains), based on given criteria, which can be based on mechanics, crystallography, etc. Hence, new grains can nucleate during primary recrystallization, with an assumed low (often zero) stored energy. Each of these new grains is described by a new level set function, which evolves subsequently according to the principles described in Section 6.1. A simple way to implement nucleation consists in building a new level set function at a specific time increment and a desired spatial position. For example, the new distance function can be chosen such that the boundary of the nucleus is spherical (3D) or circular (2D), centred around one node of the mesh. 
An illustration of this procedure is given in Figure 11, where nucleation has been numerically triggered at the node closest to the triple junction. Spontaneous growth is observed due to the difference in stored energy between the nucleus $(E=0)$ and the surrounding grains.

\subsection{Test case}

This application considers the channel die test performed on the digital aggregate described in Section 5.3. A 2D section is extracted from the 3D volume shown in Figure 6, leaving us with 24 grains with non uniform stored energy $E$ (see Figure 12) and stored energy gradient $\operatorname{grad}(E)$.

The considered digital experiments consist in following the evolution of the recrystallized volume fraction $X$ as a function of time $t$, for different nucleation criteria, and kinetic relations. Five experiments are considered : (A) all 1000 nuclei start growing from $t=0$ (site-saturated conditions) with random positions and a uniform constant velocity, (B) nuclei appear with Low Probability in Time (LPT), i.e. $2 \cdot 10^{-4}$, at random positions, and they grow with a uniform, constant velocity, (C) nuclei appear with LPT at random positions, but they grow according to the kinetic relation (28), (D) nuclei appear in LPT and only at the grain boundaries, growth follows (28), (E) nuclei appear in LPT and only at the maxima of $\operatorname{grad}(E)$, growth follows (28).

To compare the results of these experiments, reference is made to the JMAK theory [47-49] predicting the recrystallized fraction $X$ as a function of the annealing time $t$ :

$$
X=1-\exp \left(-\beta t^{n}\right)
$$

In this equation $\beta$ and $n$ are constant, and $n$ is referred to as the JMAK exponent. Assuming a two-dimensional growth, the JMAK theory predicts $n=2$ for site-saturated conditions, and $n=3$ for a low constant nucleation rate. Deviations from these $n$ values and from the linear JMAK kinetics have often been observed experimentally, e.g. [50,51]. A linear kinetics refers to a constant value of $n$, i.e. a linear JMAK plot displaying $\ln [-\ln (1-X)]$ as a function of $\ln (t)$. In experiments (A) to (E), a least-square regression analysis on the numerical data is performed, providing values of $n$. Experiments (A) and (B) provide very accurate linear JMAK plots with values of $n=2$ and 3, respectively. This validates the digital experiment set up. Figures 13 and 14 then show the results of experiments (C), (D) and (E). In each 
experiment, a single value of $n$ does not allow fitting the numerical results with sufficient accuracy. It can be noticed that the non uniform stored energy in the aggregate is only responsible for a small deviation from a linear JMAK plot (case C), while the spatial positioning of the nuclei leads to stronger deviations (cases D and E). These results must be placed in the context of repeated discussions in the literature on the reasons of deviations from linear JMAK plots, where several reasons for such deviations have been put forward : heterogeneous distribution of stored energy [50,52], competition between recovery and recrystallization [51,53], anisotropic growth [54,55] due to anisotropic values of mobility $m$ as a function of the crystallographic nature of boundaries, spatial and time distribution of nuclei [55,52]. The present framework has the capability of systematically investigating all these effects, which are expected to have different relative contributions to the overall recrystallization kinetics, depending on the type of material. For example, the heterogeneity of stored energy is expected to increase for metals with hexagonal compact (hcp) lattice, compared to those with cubic crystal symmetries. Experiment $\mathrm{C}$ for hcp metals might therefore lead to much stronger deviations from a linear kinetics than the one observed in Figure 14, which is only applicable to FCC metals.

\section{Conclusions}

In this paper, the use of digital microstructures is illustrated and first numerical developments and results are described. Conversion of digital formats into finite element meshes is facilitated by the use of a level set description of interfaces. Automatic meshing and remeshing operations rely on the values of level set functions, both for spatial localization of the interfaces and for the definition of appropriate metrics for anisotropic mesh generation. The positioning of the interfaces is updated when large plastic strain of the polycrystalline aggregate occurs, even when using automatic remeshing. Computation of the stored energy field within the aggregate, using crystal plasticity based constitutive laws, is the starting point for the subsequent modelling of primary recrystallization using a level set framework. The grain boundary network evolves directly, superposed on the mesh inherited from the digital mechanical testing. The kinetic law is based on the difference in stored strain energy across the interfaces. A special smoothing algorithm is applied to the calculated velocity field, which allows maintaining geometrical compatibility between the different grains, when combined with periodic automatic (anisotropic) remeshing. Nucleation events can be accurately triggered based on 
defined criteria, using data calculated during mechanical testing. Overall, the model is able to account for various effects related to microstructure topology, crystallographic features, and mechanical quantities, such that it can be used to decide which mechanisms are the most relevant with respect to experimental data. The selection of the most relevant mechanisms can then be used to develop homogenized, computationally cheaper models, appropriate to a defined material category.

Future work will include more realistic computations of stored energy for specific materials, and the extension of the recrystallization model to 3D aggregates using a statistical number of grains. This will lead to detailed comparisons with experiments, and with other types of model (e.g. Monte Carlo, or phase field models). The proposed approach for describing primary recrystallization could also be extended to grain growth, taking into account grain boundary energy and curvature.

\section{Acknowledgements}

LD is mandated by the National Fund for Scientific Research (FSR-FNRS, Belgium).

RL, MB, HR, HD and TC received funding from the European Commission through contract no NMP3-CT-2006-

017105 (DIGIMAT project).

\section{References}

[1]

[2]

[3]

[4]

$[5]$

[6]
M.A. Miodownik, A review of microstructural computer models used to simulate grain growth and recrystallisation in aluminium alloys, J. Light Metals 2 (2002), 125-135.

A.D. Rollett and D. Raabe, A hybrid model for mesoscopic simulation of recrystallization, Comp. Mat. Sc. 21 (2001), 69-78.

D. Raabe, Introduction of a scaleable $3 D$ cellular automaton with a probabilistic switching rule for the discrete mesoscale simulation of recrystallization phenomena, Phil. Mag A79 (1999), 2339-2358.

L.Q. Chen, A novel computer simulation technique for modeling grain growth, Scripta Metall. Mater. 32 (1995), 115-120.

H.-K. Zhao, T. Chan, B. Merriman and S. Osher, A variational level set approach to multiphase motion, J. Computational Physics 127 (1996), 179-195.

G.N. Hassold and E.A. Holm, A fast serial algorithm for the finite temperature quenched Potts model, Comput. Phys. 7 (1993), 97-107. 
A.D., Rollett, Overview of modeling and simulation of recrystallization, Progress in Mat. Sci. 42 (1997), 79-99.

J.B. Collins and H. Levine, Diffuse interface model of diffusion-limited crystal growth, Phys. Rev. B31 (1985), 6119-6122.

L.Q. Chen, Phase-field models for microstructure evolution, Ann. Rev. Mater. Res. 32 (2002), 113-140.

A. Karma, Phase-field formulation for quantitative modelling of alloy solidification, Phys. Rev. Letters 8711 (2001), art. no. 115701.

C.E. Krill, L.Q. Chen, Computer simulation of 3-D grain growth using a phase-field model, Acta Mater. 50 (2002), 3057-3073.

J.A. Sethian, Level Set methods, Cambridge University Press, 1996.

S. Osher and J.A. Sethian, Fronts propagating with curvature-dependent speed: Algorithms based on Hamilton-Jacobi formulations, J. Comp. Phys. 79 (1988), 12-49.

M. Sussman, A Level Set Approach for Computing Solutions to Incompressible Two-Phase Flow, J. Comp. Phys. 114 (1994), 146-159.

B. Merriman, J. Bence and S.J. Osher, Motion of Multiple Junctions: A Level Set Approach, J. Comp. Phys. 112 (1994), 334-363.

R.E. Logé, Y.B. Chastel, Coupling the thermal and mechanical fields to metallurgical evolutions within a finite element description of a forming process, Computer Methods in Applied Mechanics and Engineering 195 (2006), 6843-6857.

P.R. Dawson, Computational crystal plasticity, Int. J. Solids Struct. 37 (2000), 115-130.

P.R. Dawson, M.P. Miller, T.-S. Han, J. Bernier, An Accelerated Methodology for the Evaluation of Critical Properties in Polyphase Alloys, Metall. Mater. Trans. A 36 (2005), 1627-1641.

D. Raabe and R.C. Decker, Coupling of a crystal plasticity finite element model with a probabilistic cellular automaton for simulating primary static recrystallization in aluminium, Model. Simul. Mater. Sci. Eng. 8 (2000), 445-462.

$\mathrm{Ph}$. Erieau, Etude expérimentale et analyse numérique de l'influence des hétérogénéités induites par la déformation à froid sur la recristallisation primaire d'un acier IF-Ti, $\mathrm{PhD}$ thesis, Ecole Centrale de Paris, Chatenay Malabry (2003).

$\mathrm{Ph}$. Erieau and C. Rey, Modeling of deformation and rotation bands and of deformation induced grain boundaries in IF steel aggregate during large plane strain compression, Int. J. Plasticity 20 (2004), 1763-1788.

T. Baudin, A.L. Etter, Ph. Gerber, A. Samet, R. Penelle and C. Rey, Influence of thermomechanical treatments on the stored energy simulated by FEM for two low carbon steels, Materials Science Forum 495-497 (2005), Pt. 2, 1291-1296.

P. Volovitch, F. Caleyo, T. Baudin, Ph. Gerber, R. Penelle and C. Rey, Monte Carlo modeling of low carbon steel recrystallization: role of thermo-mechanical treatment and chemical composition, Materials Science Forum $495-497$ (2005), Pt. 1, 507-512.

M. Béringhier, R. Logé, L. Delannay and Y. Chastel, Strategies of transport of microstructural variables for remeshing - application to texture induced mechanical anisotropy in metals, Computer Methods in Materials Science 6 (2006), 133-139. M. Béringhier, L. Delannay, Y. Chastel and R. Logé, Using Lagrangian particles to efficiently describe microstructure evolution in metal forming - application to textureinduced mechanical anisotropy, Modelling Simul. Mater. Sci. Eng. 15 (2007), 191-204. numerical tools for the multiscale modelling of recrystallization in metals, based on a digital material framework, Computer Methods in Materials Science 7 (2007), 142-149.

D. Weygand, Y. Brechet and J. Lepinoux, A vertex simulation of grain growth in $2 D$ and $3 D$, Adv. Engng. Mater. 3 (2001), 67-71. 

Method, J. Comp. Phys. 155 (1999), 410-438. microstructure in a commercial purity aluminum, Scripta Materialia 55 (2006), 75-80. T. Coupez, Génération et Adaptation de maillage par optimisation locale, La Revue Européenne des éléments finis 9 (2000), 403-423. T. Coupez, H. Digonnet, R. Ducloux, Parallel meshing and remeshing by repartitioning, Appl. Math. Modeling 25 (2000), 153-175.

K. Mocellin, L. Fourment, T. Coupez and J.-L. Chenot, Toward large scale F.E. computation of hot forging process using iterative solvers, parallel computation and multigrid algorithms, Int. J. for Num. Meth. in Engineering 2 (2001), 473-488.

R.J. Asaro and A. Needleman, Texture development and strain hardening in rate dependent polycrystals, Acta Metall. 33 (1985), 923-953.

S.R. Kalidindi, C.A. Bronkhorst and L. Anand, Crystallographic texture evolution in bulk deformation processing of FCC metals, J. Mech. Phys. Solids 40 (1992), 537-569.

A.J. Beaudoin, P.R. Dawson, K.K. Mathur and U.F. Kocks, A hybrid finite element formulation for polycrystal plasticity with consideration of macrostructural and

microstructural linking, Int. J. Plasticity 11 (1995), 501-521.
E.B. Marin and P.R. Dawson, On modeling the elastic-plastic response of polycrystalline materials, Comput. Meth. Appl. Mech. Eng. 132 (1998), 1-21.

L. Delannay, P.J. Jacques and S.R. Kalidindi, Finite element modeling of crystal plasticity with grains shaped as truncated octahedrons, Int. J. Plasticity 22 (2006), 1879-1898.

P. Van Houtte, A comprehensive mathematical formulation of an extended Taylor-BishopHill model featuring relaxed constraints, the Renouard-Wintenberger theory and a strain rate sensitivity model, Textures microstr. 8-9 (1988), 313-350.

J.W. Hutchinson, Creep and plasticity of hexagonal polycrystals as related to single crystal slip, Metall. Trans. A8 (1969), 1465-1469.

J.-L. Chenot, L. Fourment, K. Mocellin, Numerical treatment of contact and friction in FE simulation of forming processes, J. Mater. Process. Technol. 125-126 (2002), 45-52.

G.B. Sarma, B. Radhakrishnan and T. Zacharia, Finite element simulations of cold deformation at the mesoscale, Computational Materials Science 12 (1998), 105-123.

F.J. Humphreys, M. Hatherly, Recrystallization and Related Annealing Phenomena, Pergamon $2^{\text {nd }}$ edition (2004).

T. Takaki, A. Yamanaka, Y. Higa, Y. Tomita, Phase-field model during static recrystallization based on crystal-plasticity theory, J. Computer-Aided Materials Design 14 (2007), 75-84.

G. Kugler, R. Turk, Study of the influence of initial microstructure topology on the kinetics of static recrystallization using a cellular automata model, Comput. Mater. Sci. 37 (2006), 284291.

M. Bernacki, Y. Chastel, T. Coupez, R.E. Logé, Level set framework for the numerical modelling of primary recrystallization in polycrystalline materials, Scripta Materialia 58 (2008), 1129-1132.

F. Reitich, H.M. Soner, Three-phase boundary motions under constant velocities. I: The vanishing surface tension limit, Proc. Roy. Soc. Edinburgh 126A (1996), 837-865.

W.A. Johnson, R.F. Mehl, Trans. Am. Inst. Min. Eng. 135 (1939), 416.

M. Avrami, J. Chem. Phys. 7 (1939), 1103.

A.B. Kolmogorov, Akad. Nauk SSSR, Izv. Ser. Mater. 1 (1937), 355.

M. Oyarzabal, A. Matrinez-de-Guerenu, I. Gutierrez, Effect of stored energy and recovery on the overall recrystallization kinetics of a cold rolled low carbon steel, Mater. Sci. Eng. A 485 (2008), 200-209. 
C.W. Price, Comments on the extent of simultaneous recovery during recrystallization and its effect on recrystallization kinetics, Scripta Metallurgica 23 (1989), 1273-1276.

G.J. Liao, R. Le Gall, G. Saindrenan, Experimental investigations into kinetics of recrystallisation of cold rolled nickel, Mater. Sci. Technology 14 (1998), 411-416.

H.P. Stüwe, A.F. Padilha, F. Siciliano Jr., Competition between recovery and recrystallization, Mater. Sci. Eng. A 333 (2002), 361-367.

Y.A. Andrienko, N.V. Brilliantov, P.L. Krapivsky, Phys. Rev. A 45 (1992), 2263.

F. Liu, G. Yang, Effects of anisotropic growth on the deviations from Johnson-Mehl-Avrami kinetics, Acta Materialia 55 (2007), 1629-1639. 


\section{Figure Captions}

Figure 1 : A 50 grains virtual microstructure generated with the DigiMicro software (see [26]), with four particular grains represented underneath, and the zero levels of the corresponding level set functions shown in red.

Figure 2 : Conversion of a digital sample into an initial finite element mesh, starting from a Voronoï tessellation (top) or a voxel representation (bottom).

Figure 3 : A 50 grains microstructure and the corresponding finite element mesh (68 631 nodes, 385494 elements). Anisotropic refining of the mesh is done close to the boundaries according to the $\left\{\phi_{i}, 1 \leq i \leq 50\right\}$ distance functions, as detailed in the enlarged section (bottom).

Figure 4: Anisotropic mesh constructed from a digital microstructure made of 1000 grains (2 125688 nodes, 12385889 elements).

Figure 5 : Channel die test configuration and definition of boundary conditions.

Figure 6 : Distribution of the stored energy $E$ (left) and of the gradient of the stored energy $\operatorname{grad}(E)$ (right). Illustrations include the external surfaces of the 3D volume element (top) and internal grain boundaries identified as the zero level of the $\left\{\phi_{i}, 1 \leq i \leq N_{G}\right\}$ functions (bottom).

Figure 7 : Triple junction in 2D with a stored energy $E$ lower in the grain on the right $\left(E_{1}=1\right)$ than in the two grains on the left $\left(E_{2}=E_{3}=2\right)$. Representation of the velocity field with no smoothing (left), and with smoothing (right).

Figure 8 : Effect of the mesh on the velocity field at a triple junction. Isotropic mesh (12 953 nodes, 24793 elements) on the left, and anisotropic mesh (14 686 nodes, 28950 elements) on the right.

Figure 9 : motion of a triple junction in $3 \mathrm{D}$, equivalent to the $2 \mathrm{D}$ representations of Figures 7 and 8 .

Figure 10 : microstructure evolution of a 12 grains aggregate.

Figure 11 : numerical nucleation and growth at a triple junction.

Figure 12: distribution of the stored energy $E$ in a 2D section of the 3D volume of Figure 6 .

$\underline{\text { Figure } 13}$ : time evolution of recrystallized volume fraction $X$ (in blue) for experiments $\mathrm{C}$ (top), D (middle) and $\mathrm{E}$ (bottom).

Figure 14 : JMAK approximations of numerical recrystallization kinetics in experiments C, D and $\mathrm{E}$. The JMAK exponent $n$ cannot be held constant. 

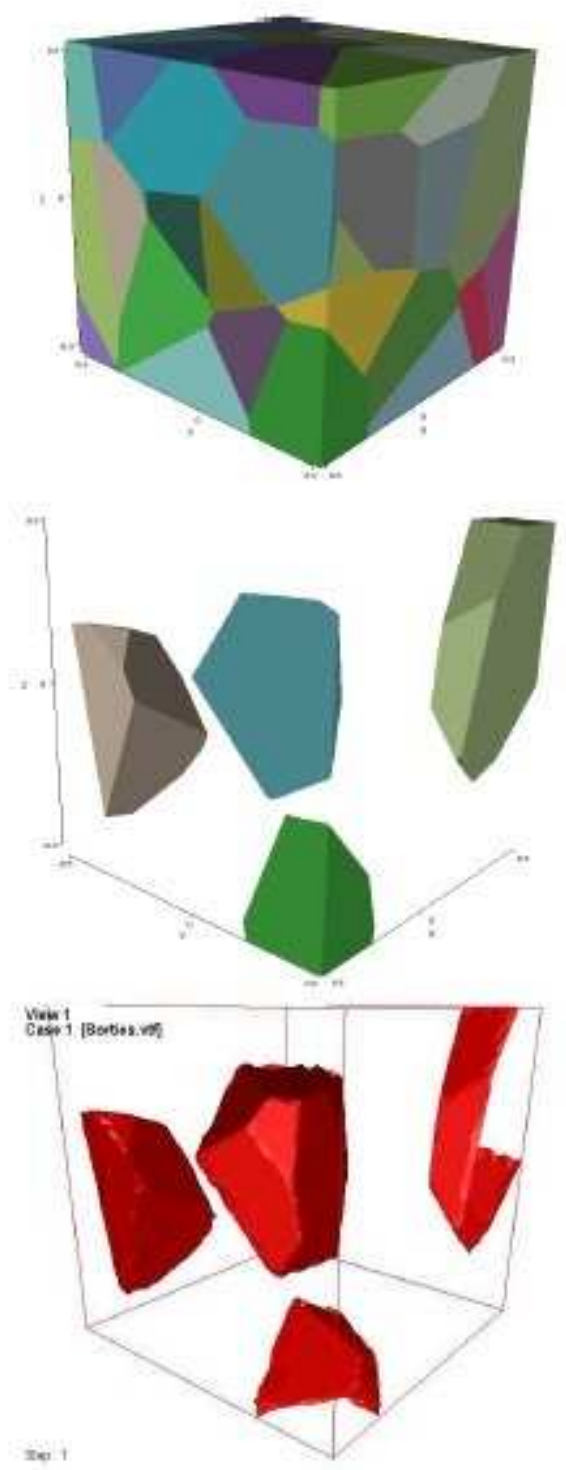

Figure 1 $72 \times 157 \mathrm{~mm}(96 \times 96 \mathrm{DPI})$ 


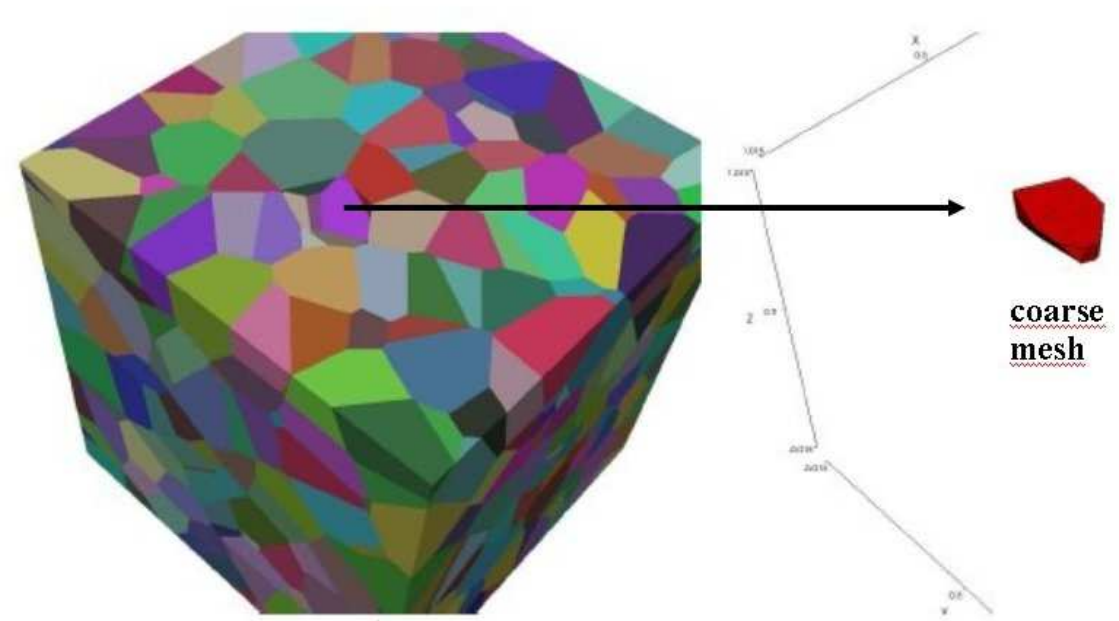

Figure 2

$236 \times 200 \mathrm{~mm}(96 \times 96 \mathrm{DPI})$

fine

mesh
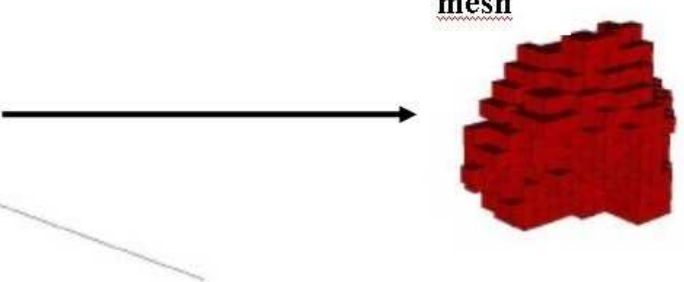

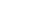



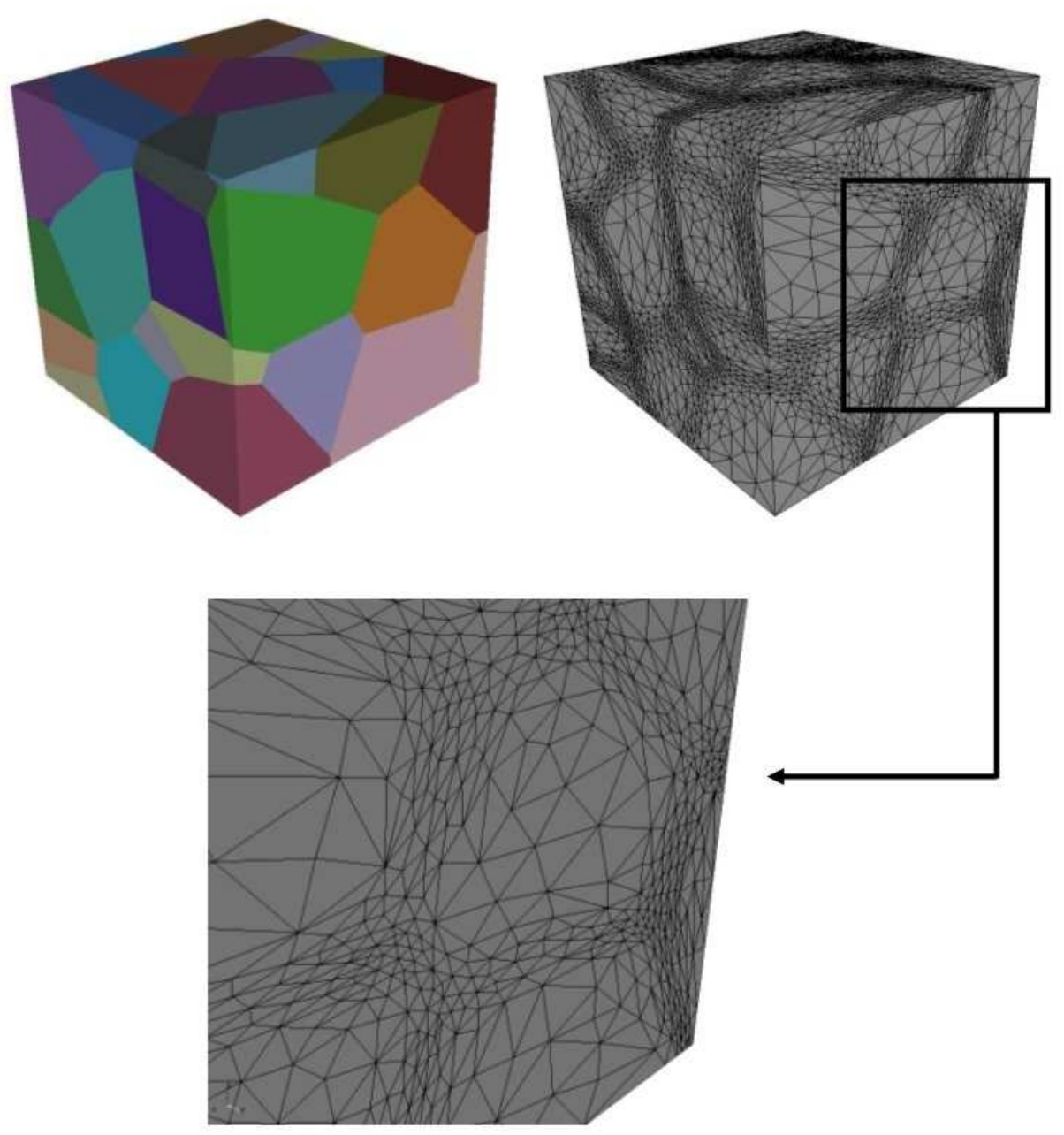

Figure 3

$211 \times 210 \mathrm{~mm}(96 \times 96 \mathrm{DPI})$ 


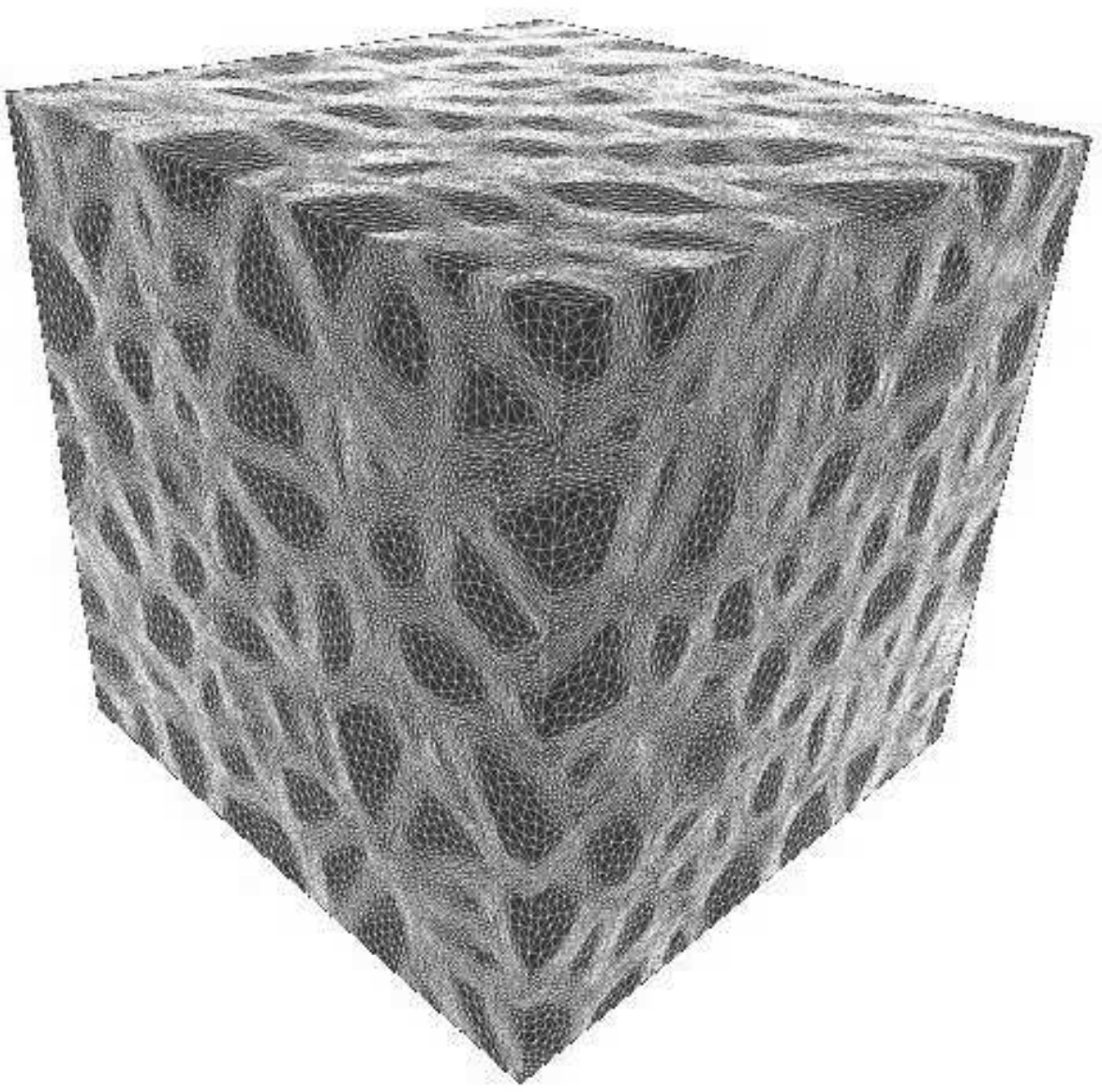

Figure 4 $182 \times 167 \mathrm{~mm}(72 \times 72 \mathrm{DPI})$ 


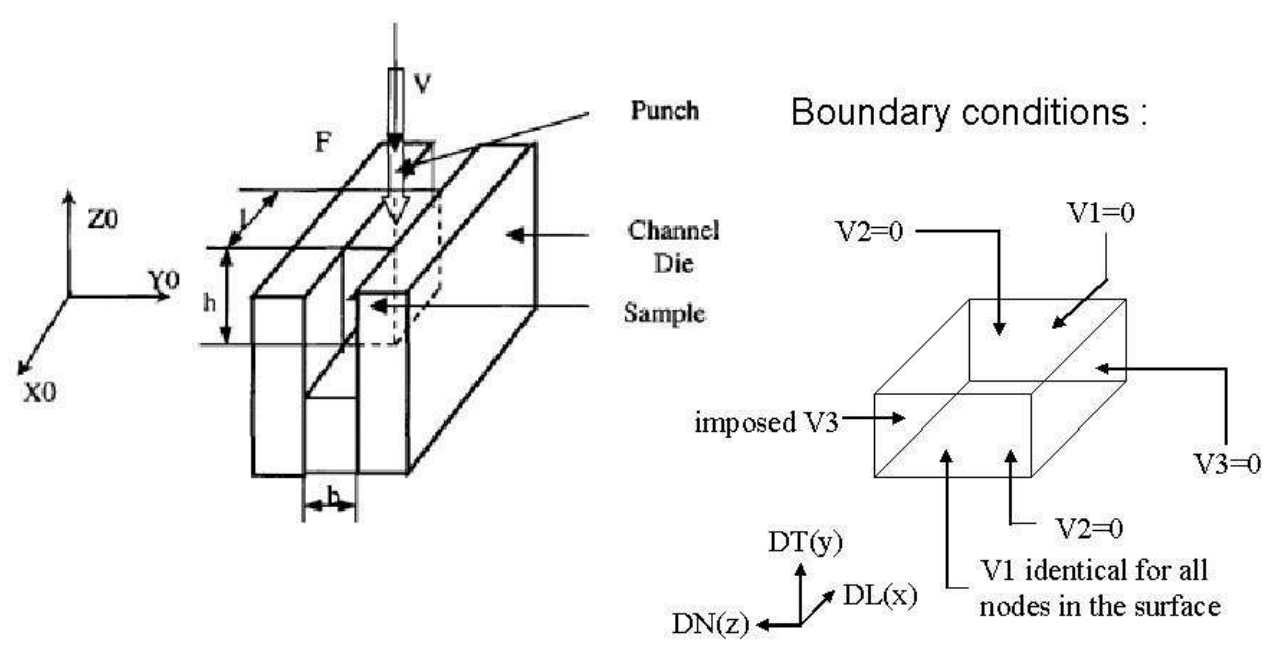

Figure 5

$319 \times 157 \mathrm{~mm}$ (72 x 72 DPI) 

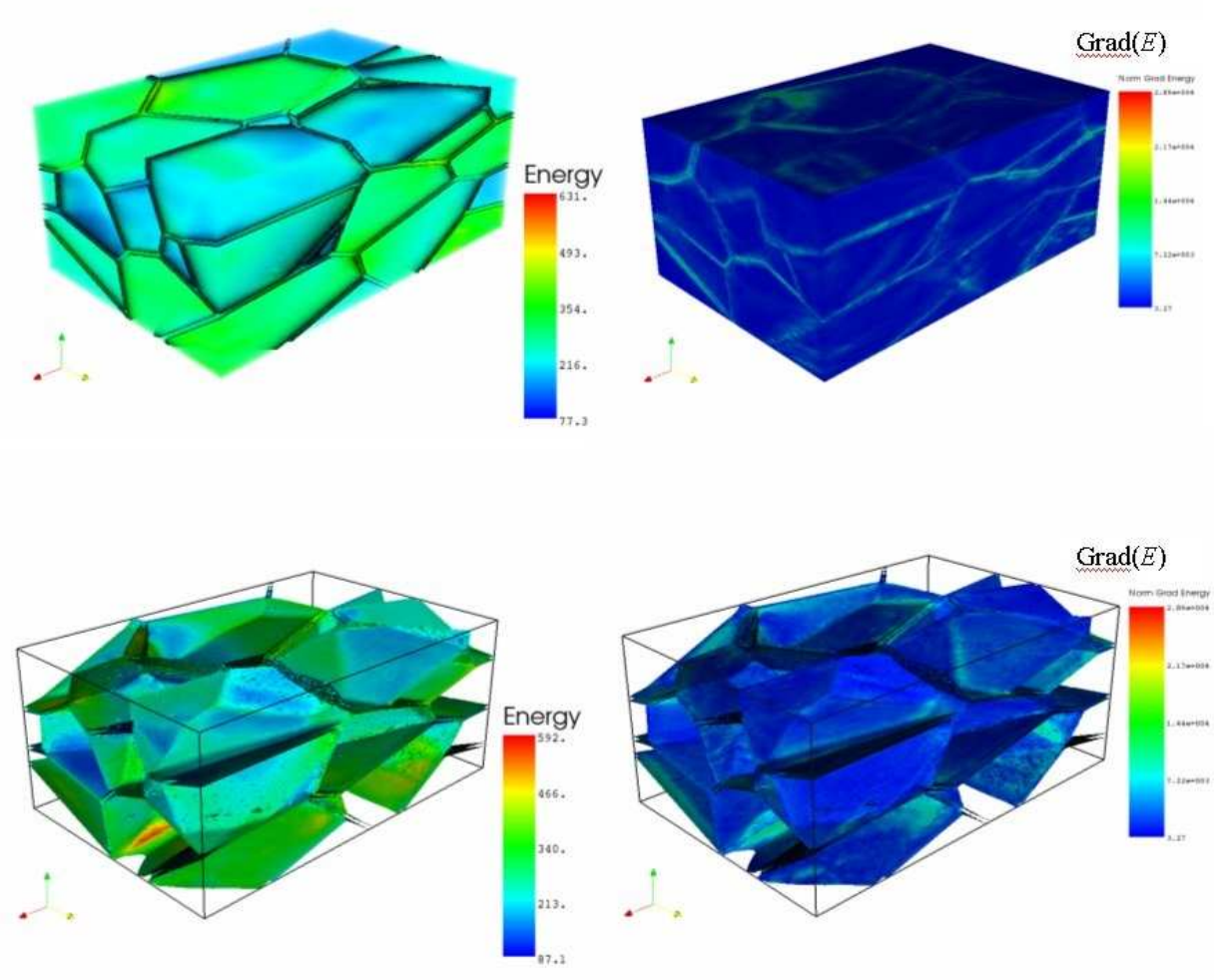

Figure 6 249x196mm (96 x 96 DPI) 


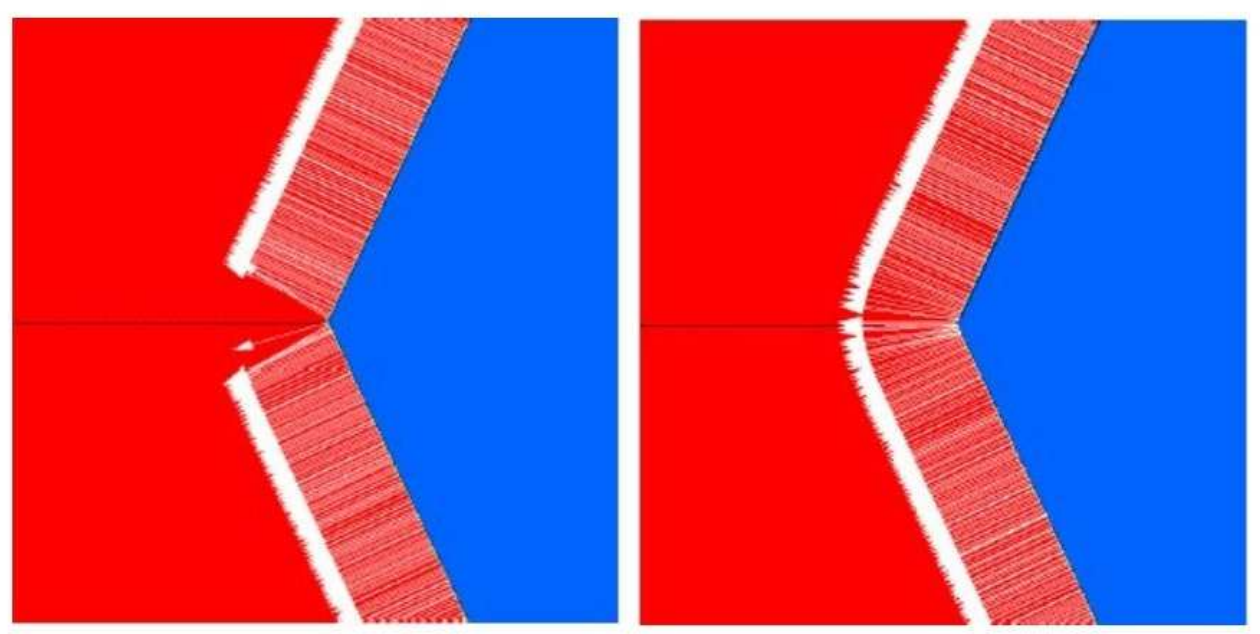

Figure 7

239x122mm (96 x 96 DPI)

http://mc.manuscriptcentral.com/pm-pml 


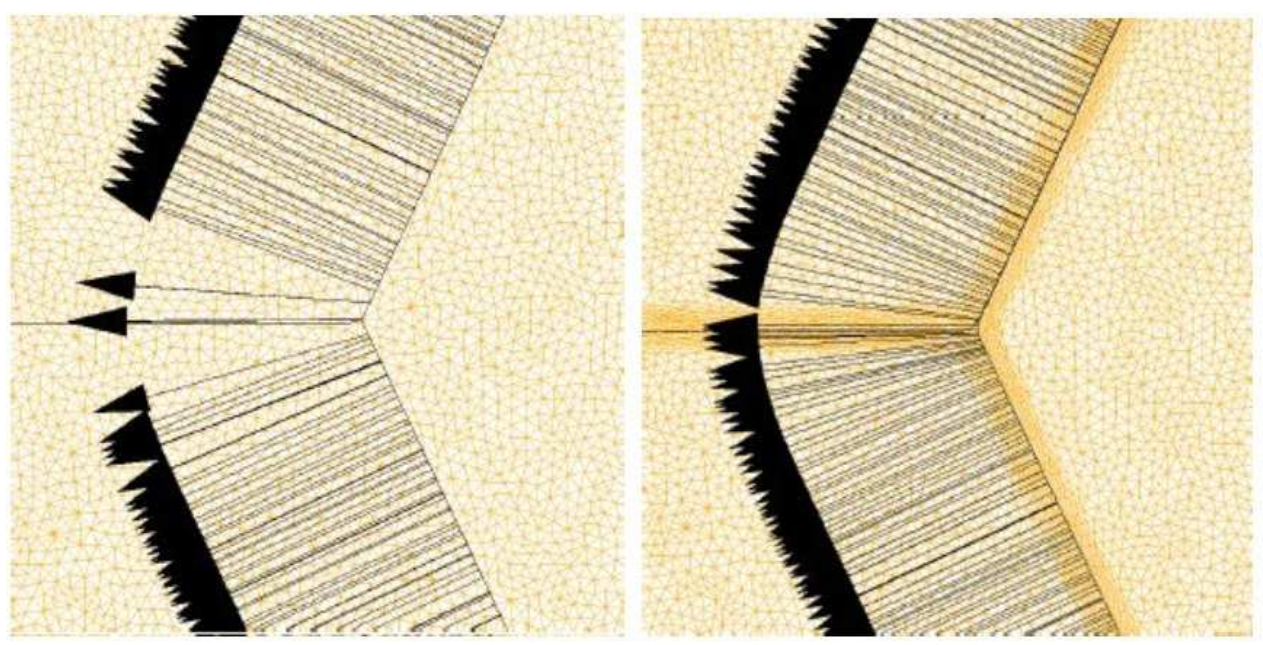

Figure 8

$237 \times 123 \mathrm{~mm}(96 \times 96 \mathrm{DPI})$ 

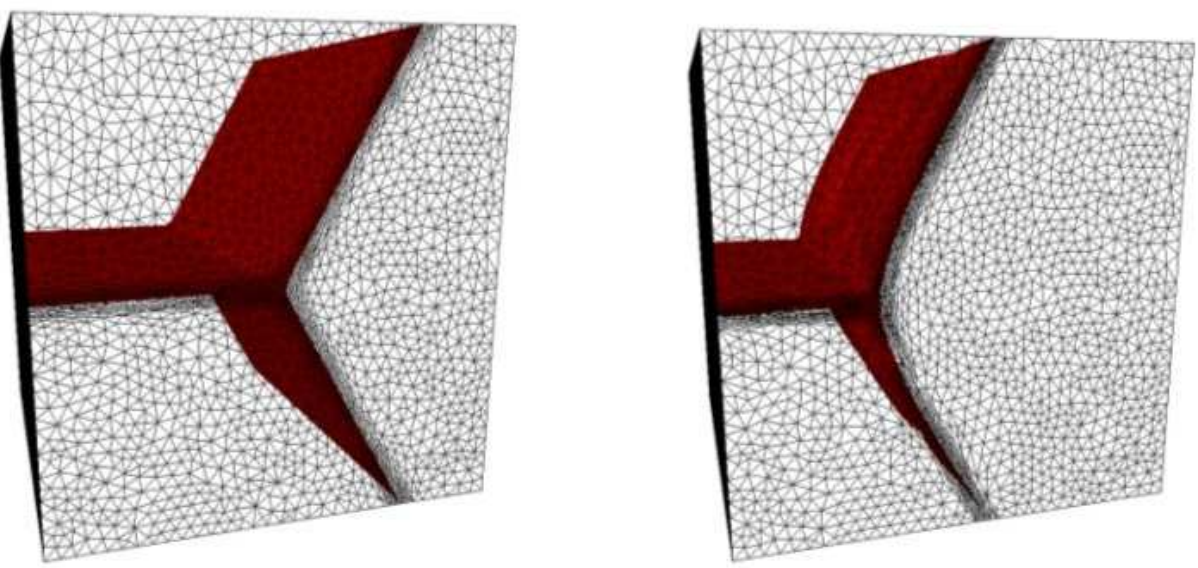

Figure 9

$200 \times 182 \mathrm{~mm}(96 \times 96$ DPI)

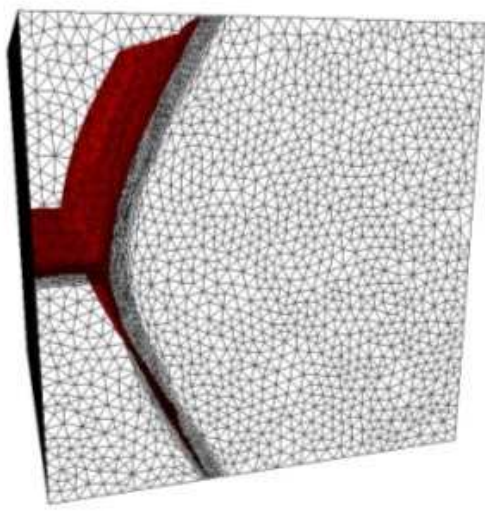



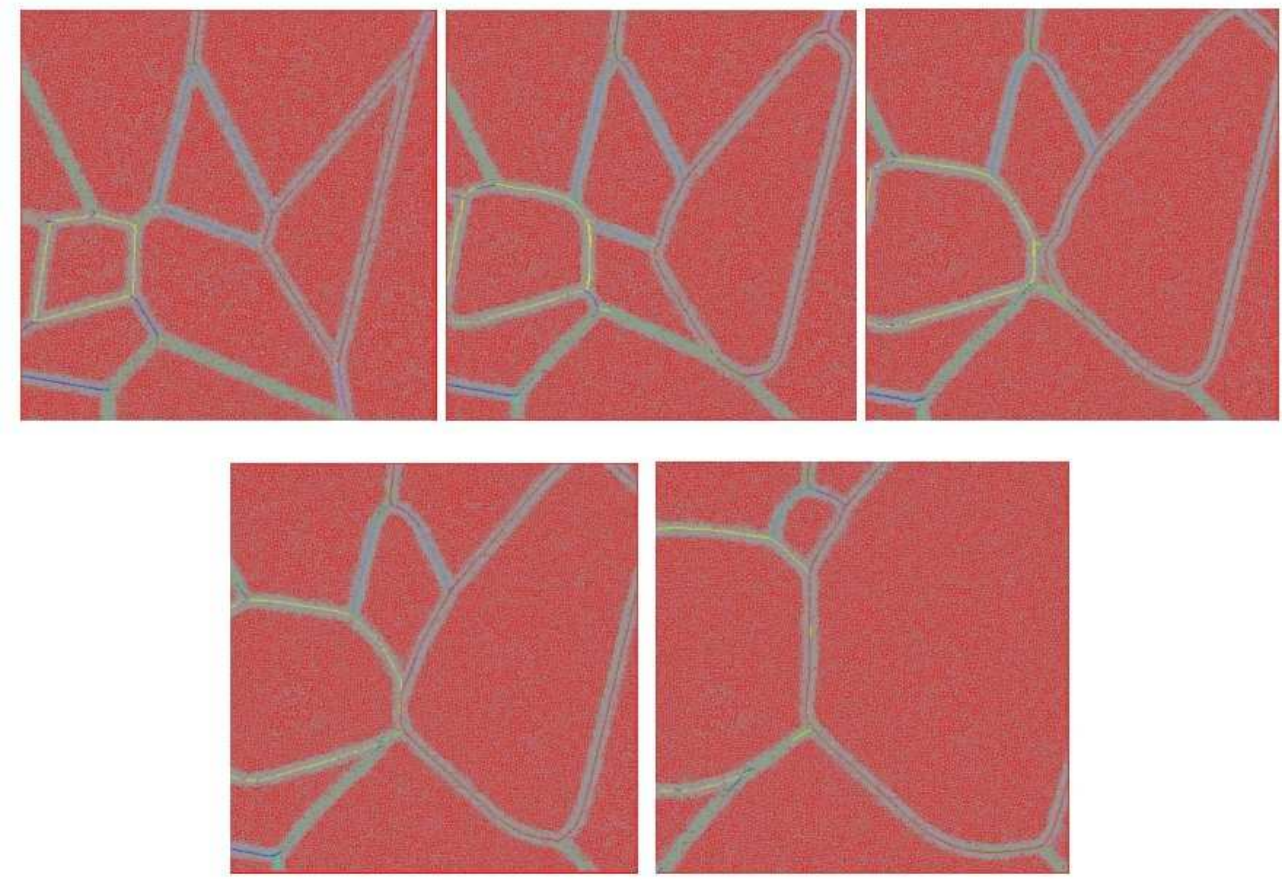

Figure 10

$236 \times 165 \mathrm{~mm}(96 \times 96 \mathrm{DPI})$ 

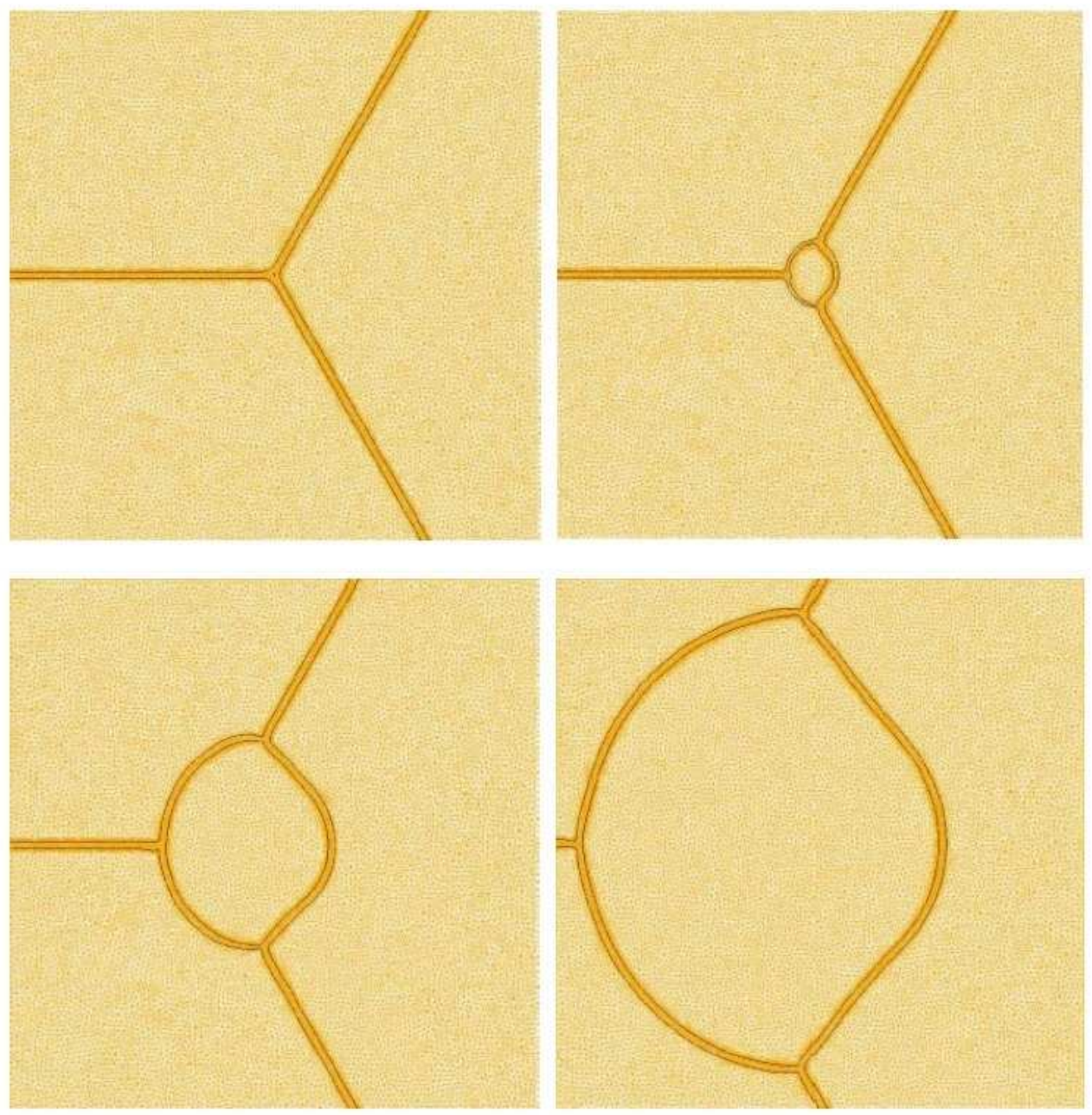

Figure 11

$203 \times 208 \mathrm{~mm}(96 \times 96$ DPI) 


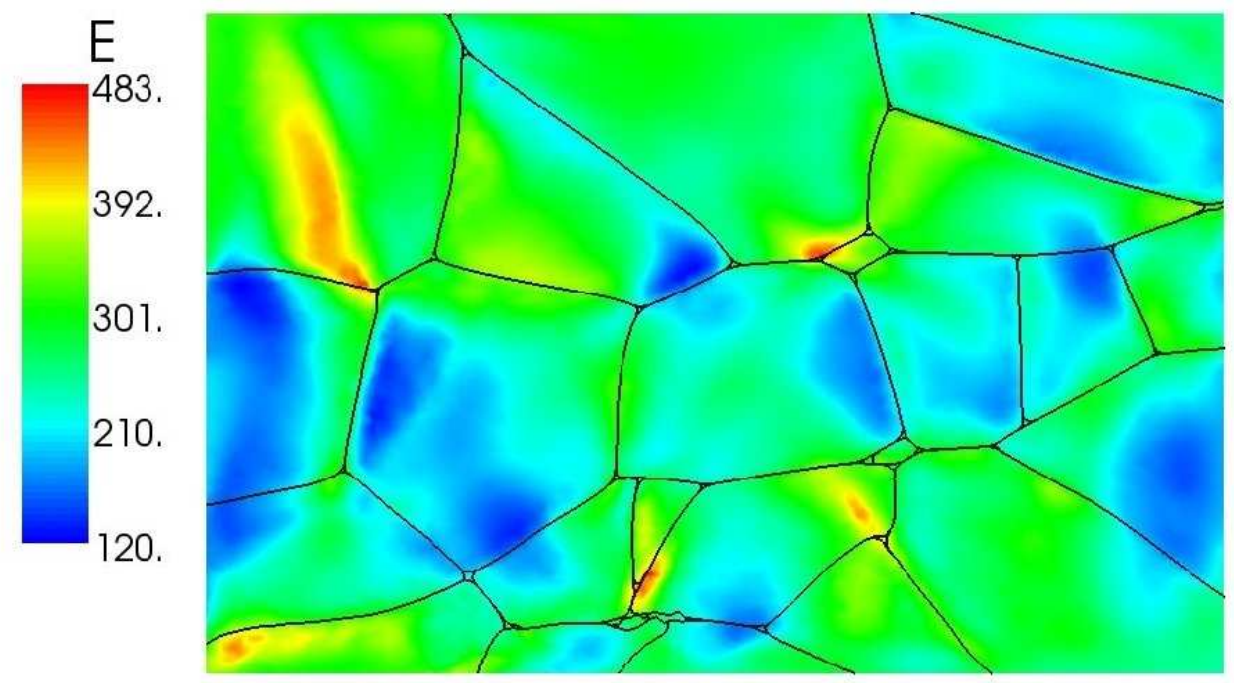

Figure 12

$248 \times 147 \mathrm{~mm}(96 \times 96 \mathrm{DPI})$ 

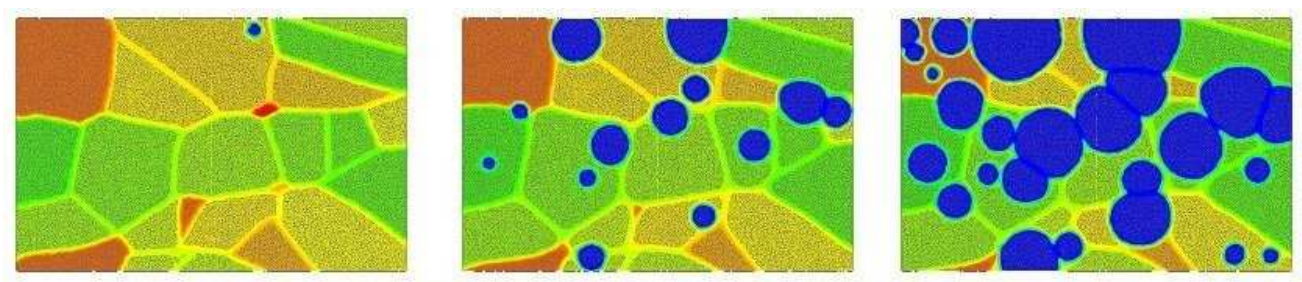

Exp C
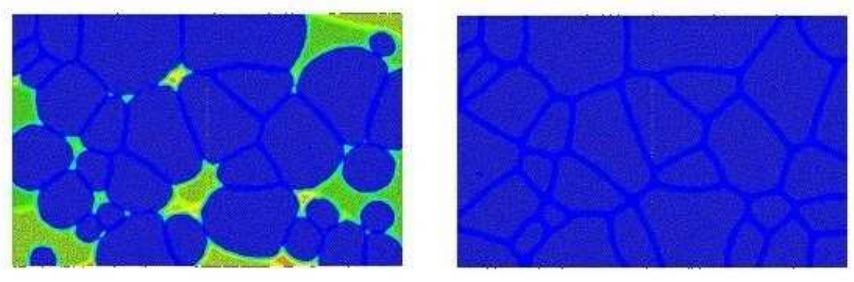

$294 \times 126 \mathrm{~mm}(72 \times 72$ DPI $)$

25

26

27

28

29

30

31

32

33

34

35

36

37

38

39

40

41

42

43

44

45

46

47

48

49

50

51

52

53

54

55

56

57

58

59

60

http://mc.manuscriptcentral.com/pm-pml 

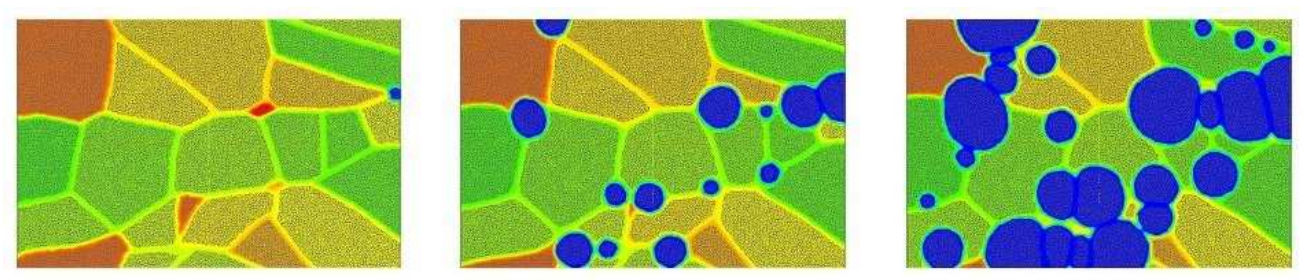

Exp D
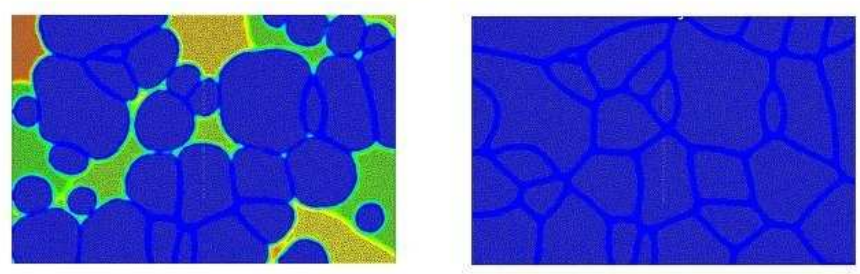

$335 \times 145 \mathrm{~mm}(72 \times 72 \mathrm{DPI})$ 

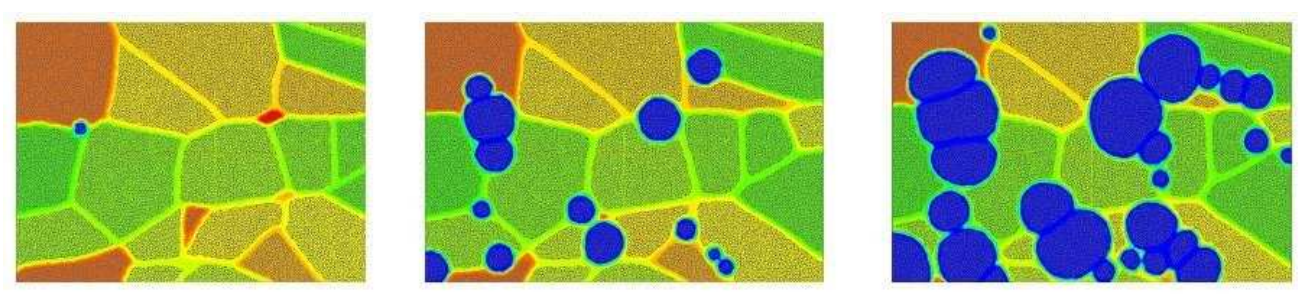

\section{$\operatorname{Exp} E$}
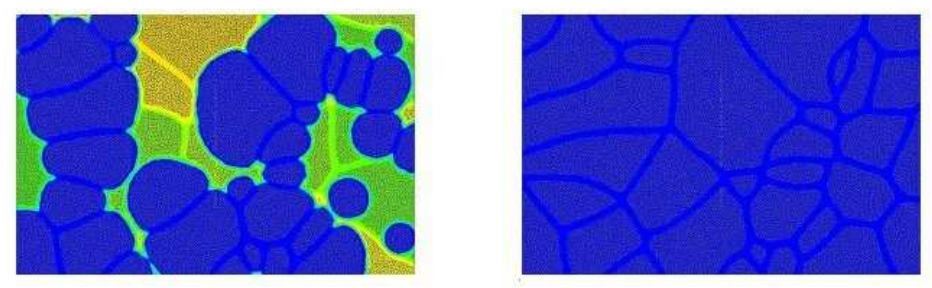

Figure 13

$317 \times 142 \mathrm{~mm}(72 \times 72 \mathrm{DPI})$

25

26

27

28

29

30

31

32

33

34

35

36

37

38

39

40

41

42

43

44

45

46

47

48

49

50

51

52

53

54

55

56

57

58

59

60

http://mc.manuscriptcentral.com/pm-pml 


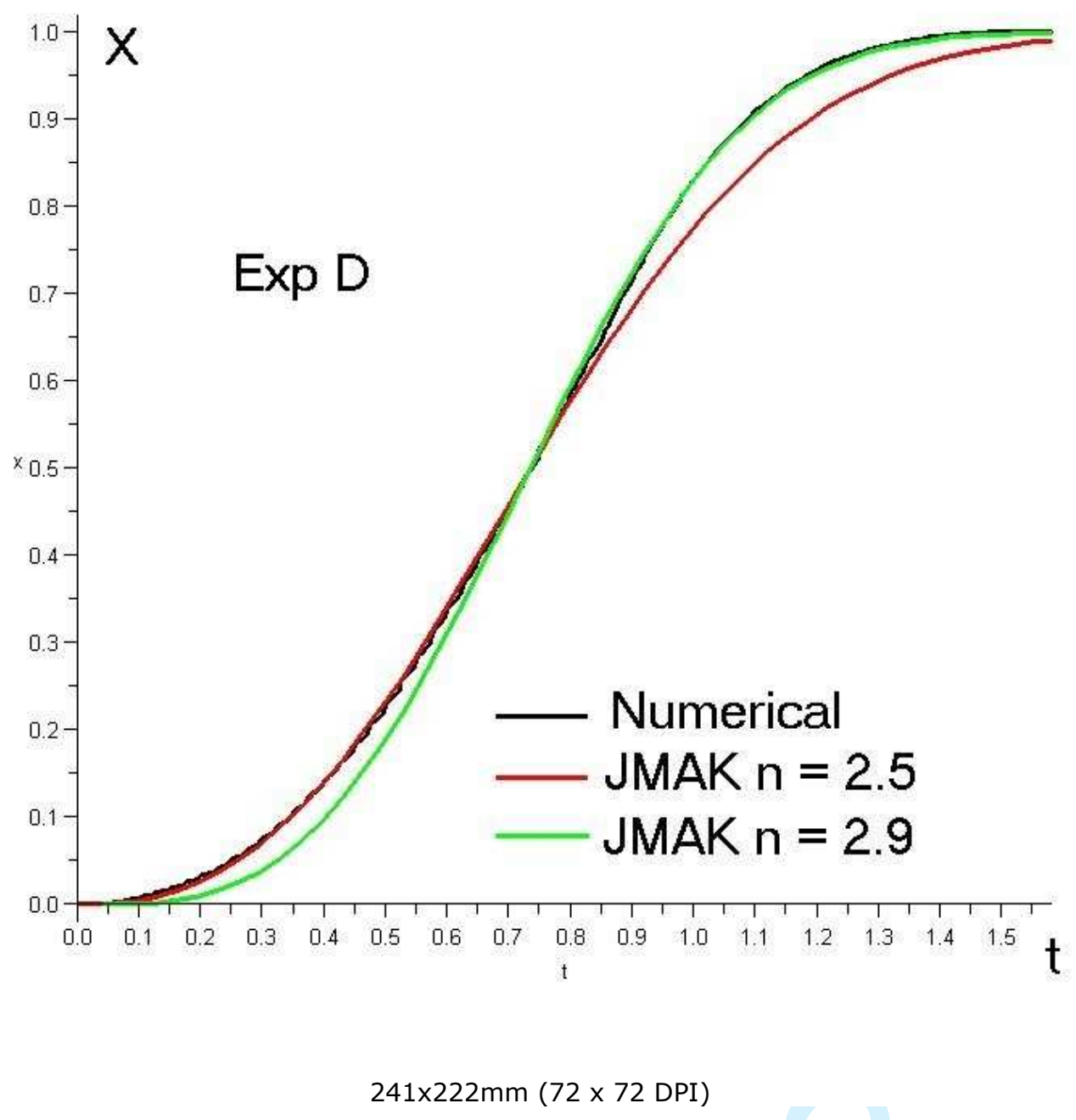

http://mc.manuscriptcentral.com/pm-pml 


1
2
3
4
5
6
7
8
9
10
11
12
13
14
15
16
17
18
19
20
21
22
23
24
25
26
27
28
29
30
31
32
33
34
35
36
37
38
39
40
41
42
43
44
45
46
47
48
49
50
51
52
53
54
55
56
57
58
60

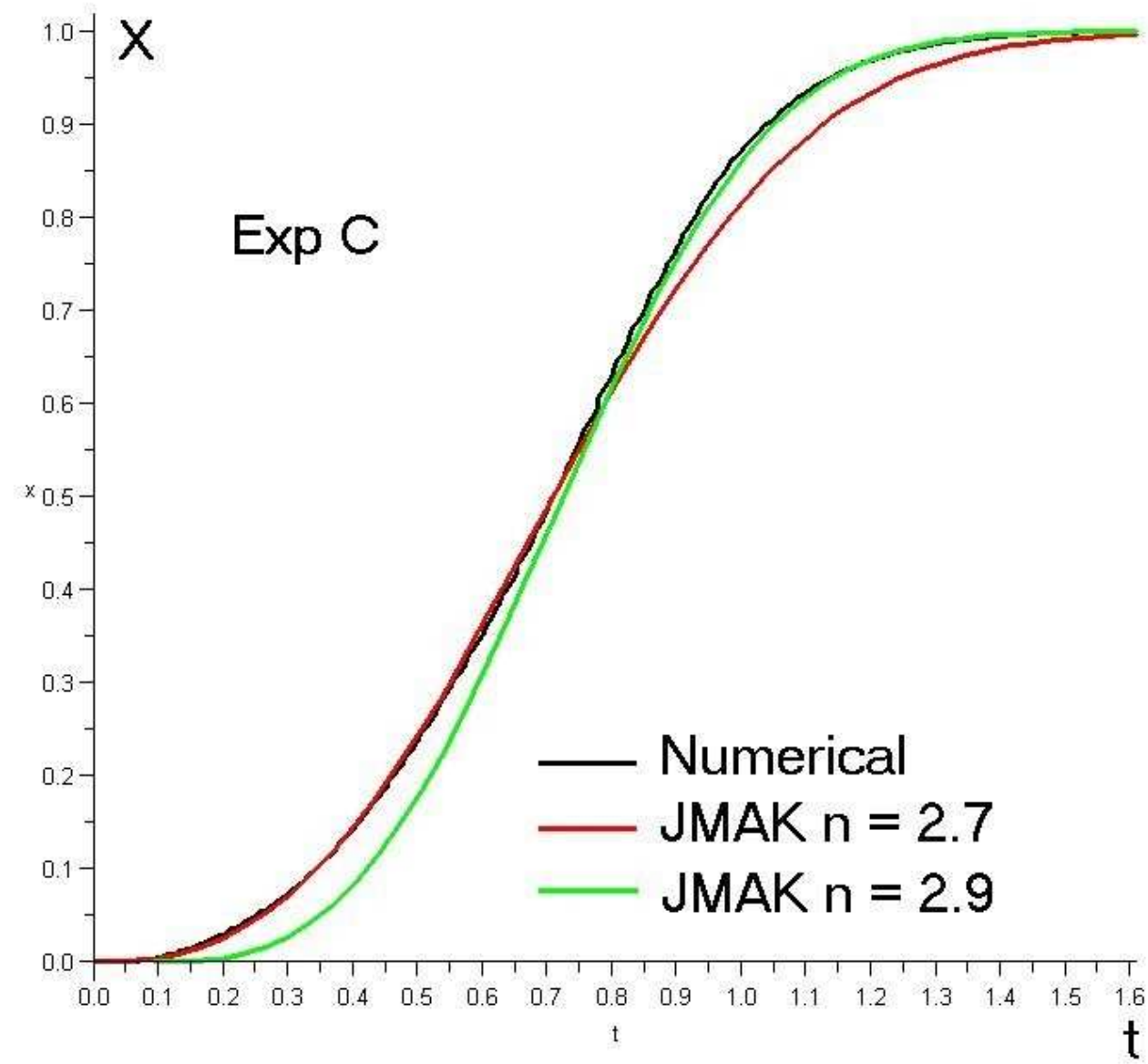

$242 \times 222 \mathrm{~mm}(72 \times 72$ DPI $)$ 


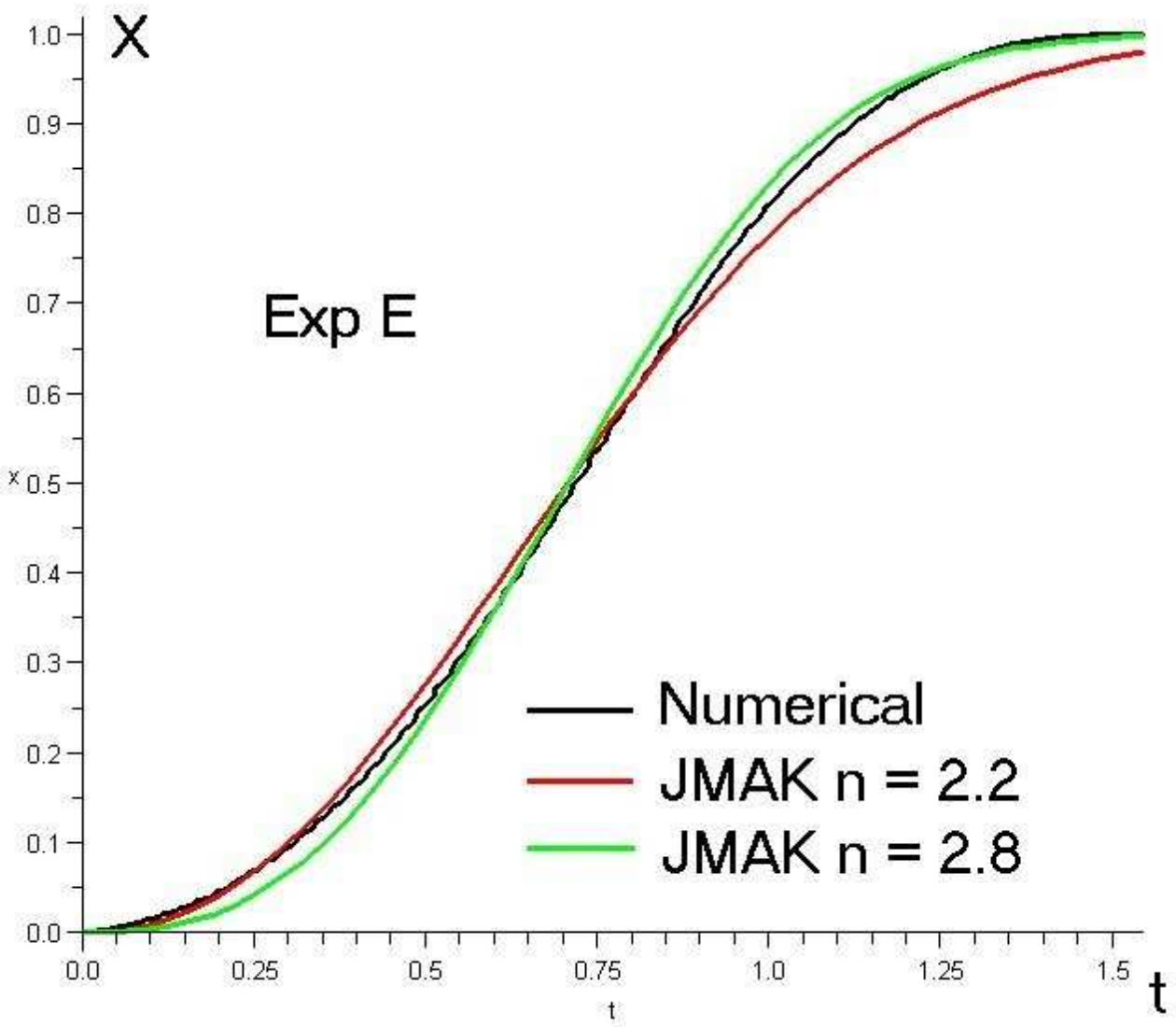

Figure 14

$230 \times 201 \mathrm{~mm}(72 \times 72 \mathrm{DPI})$ 


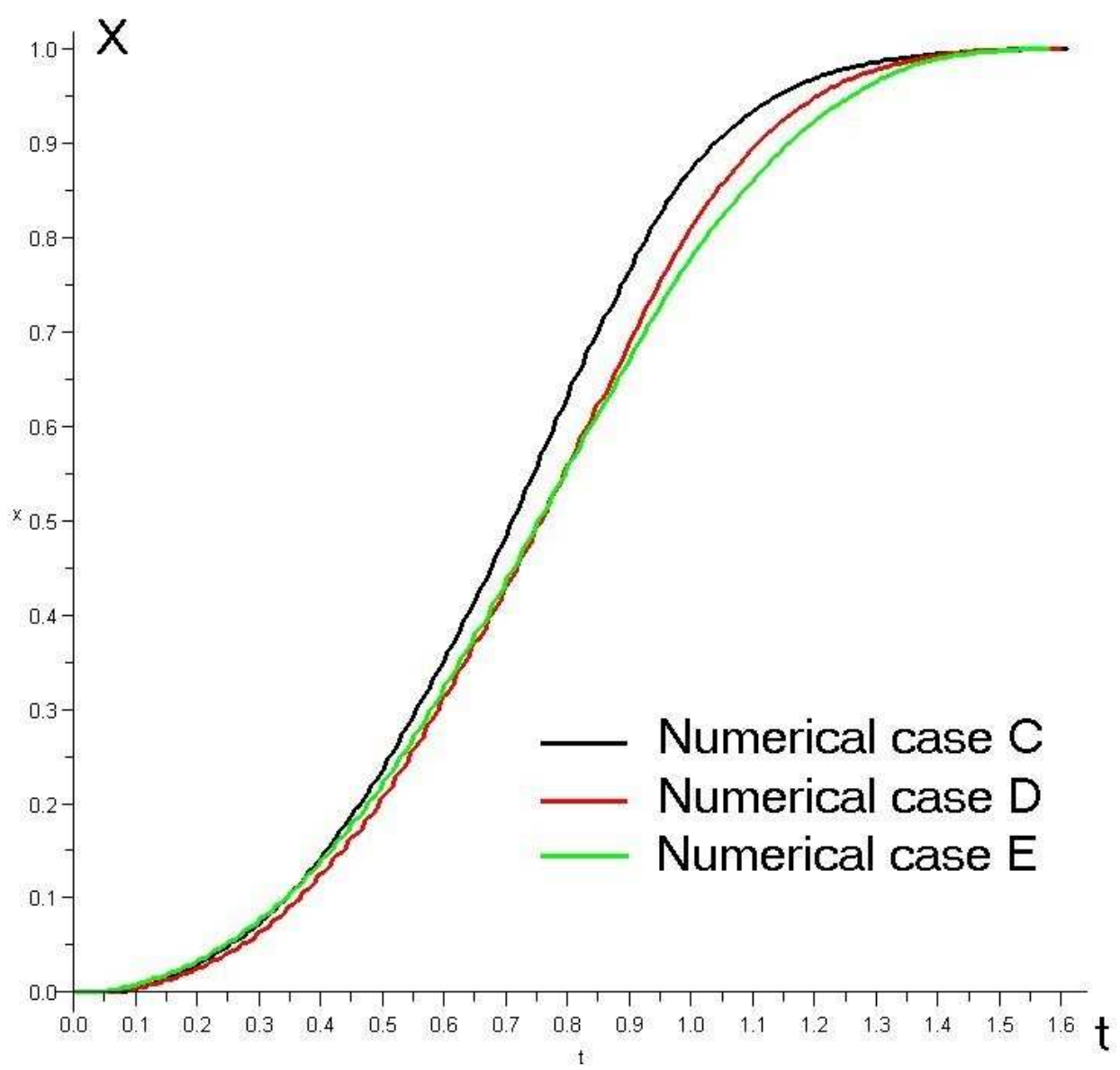

Figure 14

$271 \times 254 \mathrm{~mm}(72 \times 72 \mathrm{DPI})$ 